\title{
On the $\ell$-adic cohomology of varieties over number fields and its Galois cohomology
}

UWE JANNSEN

If $X$ is a smooth, projective variety over a number field $k$, then the absolute Galois group $G_{k}=\mathrm{Gal}(\bar{k} / k)$ acts on the étale cohomology groups $H^{i}\left(\bar{X}, Q_{\ell} / \mathbb{Z}_{\ell}(n)\right)$, where $\bar{X}=X \times_{k} \bar{k}$ for an algebraic closure $\bar{k}$ of $k$. In this paper I study some properties of these $G_{k}$-modules; in particular, I am interested in the corank of the Galois cohomology groups

$$
H^{\nu}\left(G_{k}, H^{i}\left(\bar{X}, Q_{\ell} / \mathbb{Z}_{\ell}(n)\right)\right)
$$

It turns out that this question reduces to the situation where $G_{k}$ is replaced by its decomposition groups $G_{p}$, or its maximal $S$-ramified quotient $G_{S}$ for some suitable (finite) set $S$ of places of $k$. In the last case, there are only very few results, while in the local case the situation is somewhat better, especially for the places $\mathfrak{p}$ of good reduction, but the general question is unsolved here, too. The aim of this article is to collect the known results and to discuss several conjectures - some well-known and some new which would imply that the groups in question behave rather nicely.

The basic conjecture, Conjecture 1 , is that $H^{2}\left(G_{S}, H^{i}\left(\bar{X}, Q_{\ell} / \mathbb{Z}_{\ell}(n)\right)\right)$ is finite for $i+1<n$ or $i+1>2 n$. Since the Euler-Poincaré characteristic of $G_{S}$ is well-known, this amounts to predicting a very simple number for the corank of $H^{1}\left(G_{S}, H^{i}\left(\bar{X}, Q_{\ell} / \mathbb{Z}_{\ell}(n)\right)\right)$ in this range.

In $\S 2$ I show how this conjecture for $i+1<n$ is motivated by Conjecture 2, which concerns Chern class maps from algebraic $K$-theory to $\ell$-adic cohomology and can be seen as a certain $\ell$-adic analogue of a part of Beilinson's conjectures. In fact, by results of Thomason one can draw a direct, connection to Beilinson's conjectures.

Another motivation for Conjecture 1 is that its function field analogue can be proved by using Deligne's proof of the Weil conjectures, see $\S 4$. Behind many results and conjectures of this paper stands the observation that the Weil conjectures and the theory of weights can also be used heavily in the number field case, and the hope that there might be an even closer analogy with the function field case than visible today. As an example for the first 
statement, a vanishing theorem of Serre implies that the localization map) of continuous cohomology

$$
H^{1}\left(G_{k}, M\right) \rightarrow \prod_{\mathfrak{p}} H^{1}\left(G_{\mathfrak{p}}, M\right)
$$

is injective for a torsion-free $\mathbb{Z}_{\ell}$-module $M$, which is a pure $G_{k}$-module of weight $\neq 0$.

This reduces the determination of corank $H^{2}\left(G_{k}, H^{i}\left(\bar{X}, \mathbb{Q}_{\ell} / \mathbb{Z}_{\ell}(n)\right)\right)$ to a purely local question, and leads to Conjecture 3 (sharpened form), saying that

$$
H^{i}\left(\bar{X}, Q_{\ell}(m)\right)^{G_{\mathrm{p}}}
$$

vanishes except possibly for $0 \leq m \leq \frac{i}{2}$, see $\S 5$. For $\mathfrak{p} \nmid \ell$ and good reduction, this is known, again by the Weil conjectures. For $\mathfrak{p} \nmid \ell$ and bad reduction this would follow from a well-known conjecture about the monodromy filtration, and for $\mathfrak{p} \mid \ell$ and good reduction it follows from the so-called crystalline conjecture, which has partially been proved by Fontaine-Messing and Faltings. I explain why a naive form of the monodromy filtration cannot exist for $\mathfrak{p} \mid \ell$ (this was observed by Mazur, Tate, and Teitelbaum several years ago), and I discuss what the correct analogue should be, proposing some kind of crystalline conjecture for the case of bad reduction.

If one tries to investigate whether $H^{2}\left(G_{S}, H^{i}\left(\bar{X}, Q_{\ell} / \mathbb{Z}_{\ell}(n)\right)\right)$ at least is finite for almost all $n \in \mathbb{Z}$, one is immediately led to the question of whether a certain Iwasawa module $Z$ is a $\Lambda$-torsion module, see $\S 3$. This would follow from Iwasawa's conjecture on the vanishing of the $\mu$-invariant, but is unproved yet. I show that the $\Lambda$-rank of $Z$ has to be added to Soule's expression for the corank of $H^{i}\left(X, Q_{\ell} / \mathbb{Z}_{\ell}(n)\right.$ ) (see [Sou4, Theorem 1]), whose determination is closely related to our subject. In fact, this paper can be seen as a successor of [Sou4], trying to investigate more closely the mentioned coranks and to predict precisely the range where one expects a simple expression for them.

In $\S 6$ I briefly discuss the "unstable range" $n \leq i+1 \leq 2 n$, and in $\S 7$ I treat the case of abelian varieties, where one can at least prove the local conjectures.

This paper was initiated by the investigation of $\ell$-adic Chern classes in [J3] and was influenced much by the work and ideas of Beilinson and Deligne, and by the study of Soule's paper [Sou4]. I would like to thank W. Raskind, J. M. Fontaine, W. Messing, and especially K. Wingberg, 
P. Schneider and K. Kato for valuable discussions. Finally I thank the DFG and the Mathematical Sciences Research Institute, Berkeley, for financial support and hospitality during part of this work.

\section{The basic conjecture.}

The following notations will be valid for the whole paper:

$k$ is a number field with algebraic closure $\bar{k}$,

$G_{k}=\operatorname{Gal}(\bar{k} / k)$ is the absolute Galois group of $k$,

$G_{\mathfrak{p}} \subset G_{k}$ or $G_{v} \subset G_{k}$ is a decomposition group for the prime $\mathfrak{p}$ or the place $v$ of $k$ (well defined up to conjugacy),

$X$ is a smooth, projective variety of pure dimension $d$ over $k$,

$\ell$ is a prime,

$S$ is a finite set of places of $k$, containing all places above $\infty$ and $\ell$, and all primes where $X$ has bad reduction,

$G_{S}=\operatorname{Gal}\left(k_{S} / k\right)$ is the Galois group over $k$ of the maximal $S$-ramified (= unramified outside of $S$ ) extension $k_{S}$ of $k$.

Conjecture 1 . Let $\bar{X}=X \times_{k} \bar{k}$. Then

$$
H^{2}\left(G_{S}, H^{i}\left(\bar{X}, Q_{\ell}(n)\right)\right)=0 \text { if } \begin{array}{ll}
\text { a) } & i+1<n, \text { or } \\
\text { b) } \quad i+1>2 n .
\end{array}
$$

REMARK 1: Here $H^{i}\left(\bar{X}, \mathbb{Q}_{\ell}(n)\right)=H^{i}\left(\bar{X}, \mathbb{Z}_{\ell}(n)\right) \otimes_{\mathbb{Z}_{\ell}} \mathbb{Q}_{\ell}$ is étale cohomology, and it follows from the smooth and proper base change theorem that the action of $G_{k}$ on $H^{i}\left(\bar{X}, \mathbb{Z}_{\ell}(n)\right)=\lim _{\overleftarrow{r}} H^{i}\left(\bar{X}, \mathbb{Z} / \ell^{r}(n)\right)$ is unramified outside $S$, i.e., factors through $G_{S}$. The sheaves $\mathbb{Z} / \ell^{r}(n)$ are defined as usual by $\mathbb{Z} / \ell^{r}(n)=\mu_{\ell^{r}}{ }^{\otimes n}$, where $\mu_{\ell^{r}}$ is the sheaf of $\ell^{r}$-th roots of unity [Mi1, p. 163]. The corresponding Galois modules are denoted by the same symbols, and there is a canonical isomorphism of $G_{k}$-modules $H^{i}\left(\bar{X}, \mathbb{Z}_{\ell}(n)\right) \cong$ $H^{i}\left(\bar{X}, \mathbb{Z}_{\ell}\right)(n)$, where the Tate twist $M(n)$ of a $\mathbb{Z}_{\ell}-G_{k}$-module is defined by $M(n)=M \otimes \mathbb{Z}_{\ell} \mathbb{Z}_{\ell}(n)$, with $\mathbb{Z}_{\ell}(n)=\lim _{\overleftarrow{r}} Z / \ell^{r}(n)$. The $G_{S}$-cohomology is taken via continuous cochains, and since $H^{i}\left(\bar{X}, \mathbb{Z}_{\ell}(n)\right)$ is finitely generated as a $\mathbb{Z}_{\ell}$-module, we have

$$
H^{\nu}\left(G_{S}, H^{i}\left(\bar{X}, \mathbb{Q}_{\ell}(n)\right)\right)=H^{\nu}\left(G_{S}, H^{i}\left(\bar{X}, \mathbb{Z}_{\ell}(n)\right)\right) \bigotimes_{\mathbb{Z}_{\ell}} \mathbb{Q}_{\ell}
$$

compare [J1, 5.15.c]. Throughout the paper we consider profinite groups $G$ and topological $G$-modules $M$; the cohomology will always be the continuous one. If $M$ is discrete (e.g., for $H^{i}\left(\bar{X}, \mathbb{Q}_{\ell} / \mathbb{Z}_{\ell}(n)\right)=\lim _{\vec{r}} H^{i}\left(\bar{X}, \mathbb{Z} / \ell^{r}(n)\right) \cong$ 
$\left.H^{i}\left(\bar{X}, \mathbb{Q}_{\ell} / \mathbb{Z}_{\ell}\right)(n)\right)$, this is the usual cohomology, described in [Se1]. Note that we write $\mathbb{Q}_{\ell} / \mathbb{Z}_{\ell}(n)$ for $\left(\mathbb{Q}_{\ell} / \mathbb{Z}_{\ell}\right)(n)$. We shall use the following basic facts which follow from [Ta3, §2]: if $M=\lim _{\overleftarrow{r}} M_{r}$ for a projective system $\left(M_{r}\right)$ of finite $\mathbb{Z}_{\ell^{-}} G$-modules, then

$$
H^{1}(G, M)=\underset{\leftarrow}{\lim _{T}} H^{1}\left(G, M_{r}\right) .
$$

This may be false for $H^{\nu}, \nu>1$, but if $G$ satisfies the property

$$
H^{\nu}(G, A) \text { is finite for every finite } \mathbb{Z}_{\ell}-G \text {-module } A \text {, }
$$

then we have $H^{\nu}(G, M)=\lim _{\overleftarrow{r}} H^{\nu}\left(G, M_{r}\right)$ for all $\nu$. Moreorer, if $G_{r}$ satisfies $\left(F_{\ell}\right)$ and $M$ is a finitely generated $\mathbb{Z}_{\ell}$-module, then $H^{\nu}(G, M)$ is finitely generated over $\mathbb{Z}_{\ell}$ and the canonical map

$$
H^{\nu}(G, M) \otimes \mathbb{Q}_{\ell} / \mathbb{Z}_{\ell} \rightarrow H^{\nu}\left(G, M \otimes \mathbb{Q}_{\ell} / \mathbb{Z}_{\ell}\right)
$$

is an isogeny, i.e., has finite kernel and cokernel. Similarly, if $N$ is a cofinitely generated $\mathbb{Z}_{\ell}$-torsion $G$-module (i.e., the Pontrjagin dual $N^{*}$ is a finitely generated $\mathbb{Z}_{\ell}$-module), then $H^{\nu}(G, N)$ is cofinitely generated and the canonical map

$$
H^{\nu}\left(G, T_{\ell} N\right) \rightarrow T_{\ell} H^{\nu}(G, N)
$$

is an isogeny, where $T_{\ell} A=\lim _{\tau_{r}} A_{\ell^{r}}$ is the Tate module of an abelian group $A$. Again this may be false without $\left(F_{\ell}\right)$.

We fix some more notation: If $A$ is an abelian group and $r$ is a natural number, let $A / r=A / r A$ and $A_{r}=\{a \in A \mid r a=0\}$. If a group $C_{i}$ acts on $A$, let $A^{G}$ and $A_{G}$ be the fixed and cofixed module (module of coinvariants), respectively. For an $\ell$-primary torsion group $A$, let $\lim A$ be the corank of its maximal $\ell$-divisible subgroup $\ell-\operatorname{Div} A$; that is, $\operatorname{dim} A=$ $\operatorname{dim}_{F_{\ell}}(\ell-\operatorname{Div} A)_{\ell}$, where $F_{\ell^{r}}$ is the field with $\ell^{r}$ elements.

LeMma 1 . The following statements are equivalent:

a) $H^{2}\left(G_{S}, H^{i}\left(\bar{X}, Q_{\ell}(n)\right)\right)=0$.

b) $H^{2}\left(G_{S}, H^{i}\left(\bar{X}, \mathbb{Z}_{\ell}(n)\right)\right)$ is finite.

c) $H^{2}\left(G_{S}, H^{i}\left(\bar{X}, \mathbb{Q}_{\ell} / \mathbb{Z}_{\ell}(n)\right)\right.$ is finite.

d) (if $\ell \neq 2$, or if $k$ is totally imaginary) $H^{2}\left(G_{S}, \tilde{H}^{i}\left(\overline{\mathrm{X}}, \mathbb{Q}_{\ell} / \mathbb{Z}_{\ell}(n)\right)=0\right.$. where $\tilde{H}^{i}\left(\overline{\mathrm{X}}, \mathbb{Q}_{\ell} / \mathbb{Z}_{\ell}(n)\right)=\ell-\operatorname{Div} H^{i}\left(\bar{X}, Q_{\ell} / \mathbb{Z}_{\ell}(n)\right)$. 
Proof: It follows from class field theory that $G_{S}$ satisfies $\left(F_{\ell}\right)$ (cf. [Mi2, I 4.15]). Hence the groups in b) and c) are of finite and cofinite type, respectively, and the maps

$$
H^{\nu}\left(G_{S}, H^{i}\left(\bar{X}, \mathbb{Z}_{\ell}(n)\right)\right) \otimes \mathbb{Q}_{\ell} / \mathbb{Z}_{\ell} \rightarrow H^{\nu}\left(G_{S}, H^{i}\left(\bar{X}, \mathbb{Q}_{\ell} / \mathbb{Z}_{\ell}(n)\right)\right.
$$

induced by the isogeny $H^{i}\left(\bar{X}, \mathbb{Z}_{\ell}(n)\right) \otimes \mathbb{Q}_{\ell} / \mathbb{Z}_{\ell} \rightarrow H^{i}\left(\bar{X}, \mathbb{Q}_{\ell} / \mathbb{Z}_{\ell}(n)\right)$ and (2) are again isogenies. This shows the relation

$$
\begin{aligned}
\operatorname{dim}_{\mathbf{Q}_{\ell}} H\left(G_{S}, H^{i}\left(\bar{X}, \mathbb{Q}_{\ell}(n)\right)\right)=\operatorname{rank}_{\mathbb{Z}_{\ell}} & H\left(G_{S}, H^{i}\left(\bar{X}, \mathbb{Z}_{\ell}(n)\right)\right) \\
= & \operatorname{dim} H\left(G_{S}, H^{i}\left(\bar{X}, \mathbb{Q}_{\ell} / \mathbb{Z}_{\ell}(n)\right),\right.
\end{aligned}
$$

and the equivalence of a), b) and c). Under the assumption in d), $G_{S}$ has $\ell$-cohomological dimension $c d_{\ell}\left(G_{S}\right) \leq 2[\mathrm{Br}, 2.11]$. Hence for an $\ell$-divisible $G_{S}$-module $N$ the cohomology sequence associated to $0 \rightarrow N_{\ell} \rightarrow N \stackrel{\ell}{\rightarrow}$ $N \rightarrow 0$ shows that $H^{2}\left(G_{S}, N\right)$ is $\ell$-divisible. Since an $\ell$-divisible group of finite exponent is zero, the equivalence of c) and d) follows from the finiteness of $H^{i}\left(\bar{X}, \mathbb{Q}_{\ell} / \mathbb{Z}_{\ell}(n)\right) / \tilde{H}^{i}\left(\bar{X}, \mathbb{Q}_{\ell} / \mathbb{Z}_{\ell}(n)\right)$.

In the following, we shall assume $\ell \neq 2$ or $k$ totally imaginary, for simplicity. We leave it to the reader to check that everything remains true for $\ell=2$ up to finite groups (in $\S 3$ : up to $\Lambda$-torsion modules) of exponent two, which does not matter for our purposes.

Example 1 For $X=\operatorname{Spec} k$, we have a non-trivial cohomology group only for $i=0, H^{0}\left(\operatorname{Spec} \bar{k}, \mathbb{Z}_{\ell}(n)\right)=\mathbb{Z}_{\ell}(n)$, and we may take $S=S_{\ell}=\{v \mid$ $\ell \cdot \infty\}$. The conjecture then claims

$$
H^{2}\left(G_{S}, Q_{\ell} / \mathbb{Z}_{\ell}(n)\right)=0 \text { for } \quad \begin{aligned}
& \text { a) } 1<n, \text { or } \\
& \text { b) } 1>n,
\end{aligned}
$$

i.e., for $n \neq 1$. This has been conjectured by Schneider [Sch1, p. 192]. Case a) has been proved by Soulé [Sou1] via higher Chern classes on algebraic $K$-theory; see the discussion in $\S 2$. Also for general $X$ the case b) seems to be much more mysterious; here it contains the Leopoldt conjecture (the case $n=0$, see [Sch1, §7]). Concerning $n=1$, one has $\mathbb{Q}_{\ell} / \mathbb{Z}_{\ell}(1)=\mu_{\ell \infty}$, the group of all $\ell$-power roots of unity, and

$$
H^{2}\left(G_{S}, Q_{\ell} / \mathbb{Z}_{\ell}(1)\right) \cong \operatorname{Br}_{S}(k)\{\ell\} \cong Q_{\ell} / \mathbb{Z}_{\ell}^{\# S^{\prime}-1}
$$

where $S^{\prime}=S \backslash\{v \mid \infty\}$, see [Sch1, 4.2], so this group is non-trivial in general. 
Example 2 If $E$ is an elliptic curve over $k$, then $H^{0}\left(\bar{E}, \mathbb{Z}_{\ell}\right)=\mathbb{Z}_{\ell}$ and $H^{2}\left(\bar{E}, \mathbb{Z}_{\ell}\right)=\mathbb{Z}_{\ell}(-1)$ are covered by the above. For the remaining case $i=1$, we may use the Kummer sequence $0 \rightarrow \mu_{\ell^{\nu}} \rightarrow G_{m} \stackrel{\ell^{\nu}}{\rightarrow} G_{m} \rightarrow 0$ and the isomorphisms $E(\bar{k}) \cong \operatorname{Pic}^{0}(\bar{E}) \subseteq \operatorname{Pic}(\bar{E}) \cong H^{1}\left(\bar{E}, G_{m}\right)$ to identify

$$
H^{1}\left(\bar{E}, \mathbb{Z} / \ell^{\nu}(1)\right)=H^{1}\left(\bar{E}, \mu_{\ell^{\nu}}\right)=\operatorname{Pic}(\bar{E})_{\ell^{\nu}}=E(\bar{k})_{\ell^{\nu}} .
$$

Hence the conjecture here claims $(r=n-1)$

$$
H^{2}\left(G_{S}, E_{\ell \infty}(r)\right)=0 \text { for } \quad \begin{aligned}
& \text { a) } \quad 1<r, \text { or } \\
& \text { b) } \quad 0>r,
\end{aligned}
$$

i.e., for $r \neq 0,1$ (Here $E_{\ell \infty}=\cup_{r} E(\bar{k})_{\ell^{r}}$ is the coefficient system of $\ell$-power torsion points of $E$.) If $k$ is imaginary quadratic and $E / k$ has complex multiplication by $k$, this has been proved by $\mathrm{K}$. Wingberg [Wi2] for primes $\ell$ which are regular for $E$. We shall see in $\S 7$ why $r=0,1$ definitely has to be excluded (remark 7).

It will be useful to reformulate Conjecture 1 in terms of $H^{1}\left(G_{S},-\right)$, by using Tate's calculation of the Euler-Poincare characteristic of $G_{S}$. For this, note that $X$ can be regarded as a variety over $\mathbb{Q}$ via $X \rightarrow \operatorname{Spec} k \rightarrow \operatorname{Spec} \mathbb{Q}$, and then $X \times{ }_{Q} \mathbb{C}$ is a well-defined complex projective variety (non-connected for $k \neq \mathbb{Q}$ ). In particular, $X \times_{\mathbb{Q}} \mathbb{C}$ is defined over $\mathbb{R}$ and hence has a canonical involution $F_{\infty}$ (the "infinite Frobenius", see [D3, 0.2.5]) on its Betti cohomology $H^{i}\left(X \times_{\mathbb{Q}} \mathbb{C}, \mathbb{Z}\right)$. For a ring $A \subset \mathbb{C}$ and $n \in \mathbb{Z}$, define $A(n)=A \cdot(2 \pi i)^{n} \subset \mathbb{C}$, and let $\sigma=\bar{F}_{\infty}$ act on $H^{i}\left(X \times_{\mathbb{Q}} \mathbb{C}, A(n)\right)=$ $H^{i}\left(X \times_{\mathbb{Q}} \mathbb{C}, \mathbb{Z}\right) \otimes_{\mathbb{Z}} A(n)$ via $F_{\infty} \otimes c$, where $c$ is the complex conjugation. Let $H^{i}\left(X \times_{\mathbb{Q}} \mathbb{C}, A(n)\right)^{ \pm}$be the eigenspace for the eigenvalue \pm 1 of $\sigma$, respectively.

Lemma 2. If $\chi\left(G_{S}, V\right)=\sum_{\nu=0}^{3}(-1)^{\nu} \operatorname{dim}_{Q_{\ell}} H^{\nu}\left(G_{S}, V\right)$ for a Q Q $\ell^{\text {-vector }}$ space $V$ with continuous action of $G_{S}$, then

$$
-\chi\left(G_{S}, V\right)=\sum_{v \mid \infty} \operatorname{dim}_{Q_{\ell}} V-\sum_{\substack{v \mid \infty \\ v \text { real }}} \operatorname{dim}_{Q_{\ell} \ell} V^{G_{v}} .
$$

As a consequence,

$$
\chi\left(G_{S}, H^{i}\left(\bar{X}, \mathbb{Q}_{\ell}(n)\right)\right)=-\operatorname{dim}_{\mathbb{R}} H^{i}\left(X \times_{\mathbb{Q}} \mathbb{C}, \mathbb{P}(n)\right)^{-}=: \chi_{i, n}(X)=: \chi_{i, n}
$$


in particular, these numbers are independent of $\ell$ and $S$ (satisfying our assumptions) and depend only on $n \bmod 2$.

Proof: This is proved as Proposition 2 in [Sou4], cf. also [Sch1, 4.6]. Since it is important for the sequel, we sketch the argument. Let $\Lambda$ be a $G_{S^{-}}$ equivariant lattice in $V$ (i.e., a $\mathbb{Z}_{\ell}$-submodule with $\Lambda \otimes \mathbb{Z}_{\ell} \mathbb{Q}_{\ell} \cong V$ ), and let $A=V / \Lambda$. Then $\operatorname{dim}_{Q_{\ell}} H^{\nu}\left(G_{S}, V\right)=\operatorname{dim} H^{\nu}\left(G_{S}, A\right)=\operatorname{dim}_{\mathbf{F}_{\ell}} H^{\nu}\left(G_{S}, A\right)_{\ell}$ $-\operatorname{dim}_{\mathrm{F}_{\ell}} H^{\nu}\left(G_{S}, A\right) / \ell$, so the cohomology sequence for $0 \rightarrow A_{\ell} \rightarrow A \stackrel{\ell}{\rightarrow}$ $A \rightarrow 0$ shows

$$
\chi\left(G_{S}, V\right)=\chi\left(G_{S}, A_{\ell}\right)-\operatorname{dim}_{\mathrm{F}_{\ell}} H^{2}\left(G_{S}, A\right) / \ell,
$$

where $\chi\left(G_{S}, A_{\ell}\right)=\sum_{\nu=0}^{3}(-1)^{\nu} \operatorname{dim}_{F_{\ell}} H^{\nu}\left(G_{S}, A_{\ell}\right)=-\sum_{v \mid \infty} \operatorname{dim}_{F_{\ell}} A_{\ell}+$ $\sum_{v \mid \infty} \operatorname{dim}_{\mathbf{F}_{\ell}} H^{0}\left(G_{v}, A_{\ell}\right)$ by Tate [Ta1]. Since $H^{2}\left(G_{S}, A\right) / \ell \cong \oplus_{v \mid \infty} H^{2}\left(G_{v}, A\right)$, one easily deduces as in [Sou4]

$$
-\chi\left(G_{S}, V\right)=\sum_{v \mid \infty} \operatorname{dim} A-\sum_{\substack{v \mid \infty \\ v \text { real }}} \operatorname{dim} A^{G_{v}},
$$

i.e., the first formula. Since $H^{i}\left(X \times_{Q} \bar{Q}, Q_{\ell}\right) \cong \operatorname{Ind}_{G_{k}}^{G} H^{i}\left(\bar{X}, Q_{\ell}\right)$, where $\operatorname{Ind}_{G_{k}}^{G}$ denotes induction from $G_{k}$ to $G_{\mathbb{Q}}$, this formula implies

$$
\begin{aligned}
& -\chi\left(G_{S}, H^{i}\left(\bar{X}, \mathbb{Q}_{\ell}(n)\right)\right) \\
& \quad=\operatorname{dim}_{\mathbb{Q}_{\ell}} H^{i}\left(X \times_{\mathbb{Q}} \overline{\mathbf{Q}}, \mathbf{Q}_{\ell}(n)\right)-\operatorname{dim}_{Q_{\ell}} H^{i}\left(X \times_{\mathbb{Q}} \overline{\mathbf{Q}}, \mathbb{Q}_{\ell}(n)\right)^{G_{\infty},}
\end{aligned}
$$

where $G_{\infty} \subset G_{\mathbb{Q}}$ is a decomposition group at $\infty$. The choice of $G_{\infty}$ corresponds to the choice of an embedding $\tau: \overline{\mathbf{Q}} \hookrightarrow \mathbb{C}$, and via the canonical comparison isomorphism

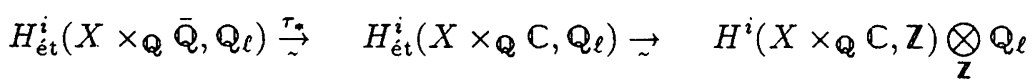

the action of $F_{\infty}$ corresponds to the action of the non-trivial element in $G_{\infty}$. Finally, the Tate twist $( \pm 1)$ on both sides changes the action of $G_{\infty}$ and $\sigma$ by a sign (i.e., by the non-trivial characters $G_{\infty} \rightarrow\{ \pm 1\},\langle\sigma\rangle \rightarrow\{ \pm 1\}$, respectively), which shows

$$
\operatorname{dim}_{\mathbb{Q}_{\ell}} H^{i}\left(X \times_{\mathbb{Q}} \overline{\mathbf{Q}}, \mathbb{Q}_{\ell}(n)\right)^{G_{\infty}}=\operatorname{dim}_{\mathbf{R}} H^{i}\left(X \times_{\mathbb{Q}} \mathbb{C}, \mathbb{R}(n)\right)^{+} .
$$

Corollary 1. a) $\operatorname{dim}_{Q_{\ell}} H^{1}\left(G_{S}, H^{i}\left(\bar{X}, Q_{\ell}(n)\right)\right) \geq-\chi_{i, n}(X)$, with equality if and only if $H^{i}\left(\bar{X}, \mathbf{Q}_{\ell}(n)\right)^{G_{S}}=0=H^{2}\left(G_{S}, H^{i}\left(\bar{X}, Q_{\ell}(n)\right)\right)$.

b) $\chi\left(G_{S}, H^{i}\left(\bar{X}, \mathbb{Q}_{\ell}(n)\right)\right)+\chi\left(G_{S}, H^{i}\left(\bar{X}, \mathbb{Q}_{\ell}(i+1-n)\right)\right)=-[k: \mathbb{Q}]$. $\operatorname{dim}_{\mathbf{Q}_{\ell}} H^{i}\left(\bar{X}, Q_{\ell}\right)$.

The fixed module $H^{i}\left(\bar{X}, Q_{\ell}(n)\right)^{G_{k}}$ is related to Tate's conjecture for $i=$ $2 n$, but it always vanishes for $i \neq 2 n$ : 
Lemma 3. If $i \neq 2 n$, then $H^{i}\left(\bar{X}, \mathbb{Q}_{\ell}(n)\right)^{G_{k}}=0=H^{i}\left(\bar{X}, \mathbf{Q}_{\ell}(n)\right)_{G_{k}}$ and $H^{i}\left(\bar{X}, Q_{\ell}(n)\right)^{G_{\mathrm{p}}}=0=H^{i}\left(\bar{X}, \mathbb{Q}_{\ell}(n)\right)_{G_{\mathrm{p}}}$ for every $\mathfrak{p} \notin S$.

Proof: Let $\mathcal{X}$ be a smooth and proper model of $X$ over $\mathcal{O}_{S}$, the ring of $S$-integers in $k$, and let $\mathcal{X}_{\mathfrak{p}}=\mathcal{X} \times_{\mathcal{O}_{S}} \kappa(\mathfrak{p})$ be its fiber at $\mathfrak{p} \in \operatorname{Spec} \mathcal{O}_{S}=$ Spec $\mathcal{O} \backslash S, \kappa(\mathfrak{p})$ denoting the residue field of $\mathfrak{p}$. By the smooth and proper base change theorem (see [Mi1, VI 4.2]), there is a canonical isomorphism (note that $\mathfrak{p} \nmid \ell$ )

$$
H^{i}\left(\bar{X}, \mathbf{Q}_{\ell}(n)\right) \cong H^{i}\left(\overline{\mathcal{X}_{\mathfrak{p}}}, \mathbf{Q}_{\ell}(n)\right),
$$

where $\overline{\mathcal{X}}_{\mathfrak{p}}=\mathcal{X}_{\mathfrak{p}} \times_{\kappa(\mathfrak{p})} \overline{\kappa(\mathfrak{p})}$ for an algebraic closure $\overline{\kappa(\mathfrak{p})}$ of $\kappa(\mathfrak{p})$. This is an isomorphism of $G_{\mathfrak{p}}$-modules via $G_{\mathfrak{p}} \rightarrow \operatorname{Gal}(\overline{\kappa(\mathfrak{p})} / \kappa(\mathfrak{p}))$, and by Deligne's proof of the Weil conjectures, $H^{i}\left(\overline{\mathcal{X}}_{\mathfrak{p}}, Q_{\ell}\right)$ is pure of weight $i$; that is, the eigenvalues of the geometric Frobenius $\operatorname{Fr}_{\mathfrak{p}} \in \operatorname{Gal}(\overline{\kappa(\mathfrak{p})} / \kappa(\mathfrak{p}))$ are algebraic integers whose archimedan absolute values are equal to $(N \mathfrak{p})^{\frac{i}{2}}$, where $N \mathfrak{p}$ is the cardinality of $\kappa(\mathfrak{p})[\mathbf{D} 4,3.3 .9]$. Hence $H^{i}\left(\overline{\mathcal{X}}_{\mathfrak{p}}, \mathbb{Q}_{\ell}\right)(n)$ is of weight $i-2 n$ $\left(\operatorname{Fr}_{\mathfrak{p}}\right.$ acts on $Q_{\ell}(1)$ via multiplication by $\left.(N \mathfrak{p})^{-1}\right)$. There can only be a non-zero fixed or cofixed module, if the eigenvalue 1 appears, that is, if the weight is zero.

\section{Connections with algebraic $K$-theory.}

Beilinson's conjectures [Be1] concern the diagram

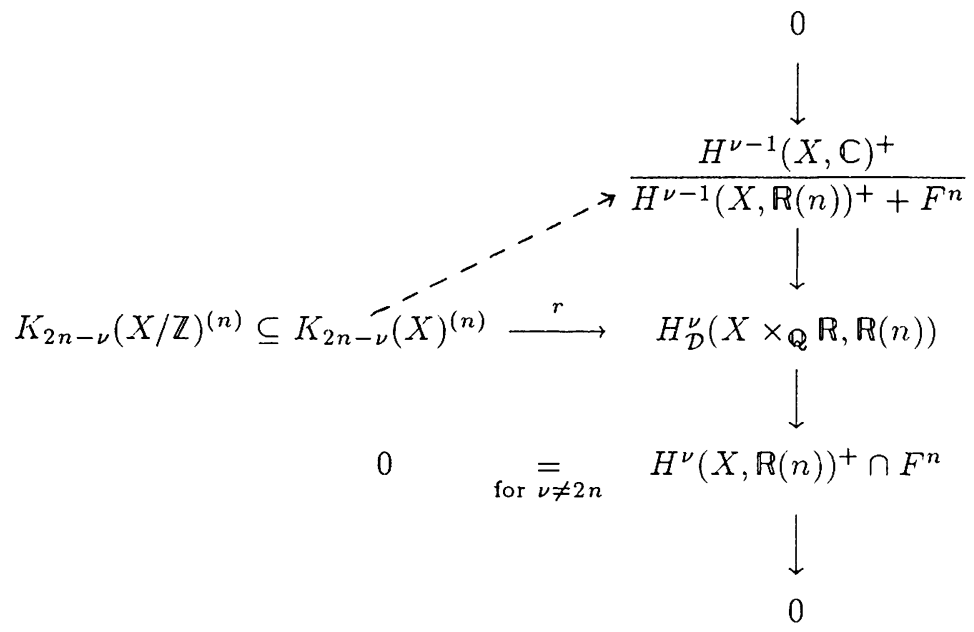

Here $H_{\mathcal{D}}^{\nu}$ denotes the Deligne cohomology, which sits in a short sequence as indicated, $H^{*}(X,-)$ stands for the Betti cohomology $H^{*}\left(X \times_{\mathbb{Q}} \mathbb{C},-\right)$, 
$F^{*}$ is the Hodge filtration on $H^{*}(X, C)$, and $K_{m}(X)^{(n)}$ is the subspace of $K_{m}(X) \otimes Q$ on which the Adams operators $\psi^{k}$ operate via multiplication by $k^{n}$. The "regulator map" $r$ is defined by Chern characters on Quillen's higher $K$-groups $K_{m}(X)$ with values in the Deligne cohomology, and factorizes as indicated for $\nu \neq 2 n$, since then $F^{n} \cap \overline{F^{n}}=H^{n, n}$ is zero. Beilinson thinks that the definition

$$
K_{m}(X / \mathbb{Z})^{(n)}=\operatorname{Im}\left(K_{m}(\mathcal{X})^{(n)} \rightarrow K_{m}(X)^{(n)}\right)
$$

$\mathcal{X}$ a flat, proper model for $X$ over $\mathbb{Z}$, does not depend on the choice of $\mathcal{X}$ and then conjectures, among other things, the following, where we set $i=\nu-1$ for convenience.

Conjecture (Beilinson). For $i<2 n-2$ the regulator map

$$
r: K_{2 n-i-1}(X / \mathbb{Z})^{(n)} \otimes \mathbb{R} \rightarrow H^{i}(X, \mathbb{C})^{+} /\left(H^{i}(X, \mathbb{R}(n))^{+}+F^{n}\right)
$$

is an isomorphism, and the dimension of these vector spaces is the order of zero of the $L$-function $L(V, s)$ for $V=\left\{H^{i}\left(\bar{X}, Q_{\ell}\right)\right\}$ at $s=m:=i+1-n$.

REMARK 2: The $L$-function of the strictly compatible system of $\ell$-adic representations $V$ is defined by the Euler product

$$
L(V, s)=\prod_{\mathfrak{p}} L_{\mathfrak{p}}(V, s)=\prod_{\mathfrak{p}} \frac{1}{\operatorname{det}\left(1-\operatorname{Fr}_{\mathfrak{p}}(N \mathfrak{p})^{-s} \mid V^{I_{\mathfrak{p}}}\right)}
$$

Here $I_{\mathfrak{p}}$ is an inertia group at the prime $\mathfrak{p}, \mathrm{Fr}_{\mathfrak{p}}$ is a geometric Frobenius at $\mathfrak{p}$, and it is conjectured that

$$
\operatorname{det}\left(1-\operatorname{Fr}_{\mathfrak{p}} T \mid V^{I_{\mathfrak{p}}}\right)=\operatorname{det}\left(1-\operatorname{Fr}_{\mathfrak{p}} T \mid H^{i}\left(\bar{X}, Q_{\ell}\right)^{I_{\mathfrak{p}}}\right), \mathfrak{p} \nmid \ell,
$$

lies in $\mathbb{Z}[T]$, is independent of $\ell$ (with $\mathfrak{p} \nmid \ell$ ), and that $L(V, s)$ is convergent for $\operatorname{Re}(s) \geq \frac{i}{2}+1$. By Deligne's proof of the Weil conjectures [D4], all this is known if one omits the factors where $X$ has bad reduction. For the consideration of $m=i+1-n<\frac{i}{2}$, one has to assume meromorphic continuation of $L(V, s)$. The conjectured analytic behavior of $L(V, s)$ (see [D3]) is roughly described by the following picture.

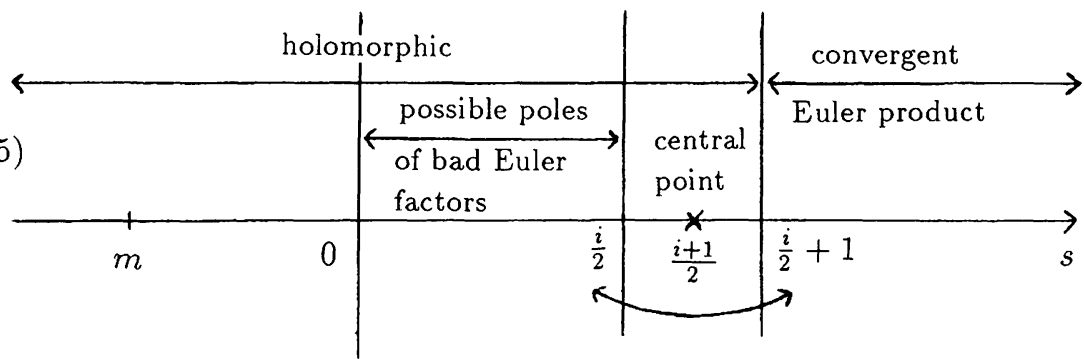

functional equation $s \leftrightarrow i+1-s$ 
The equality

$$
\underset{s=m}{\operatorname{ord}} L(V, s)=\operatorname{dim}_{\mathbf{R}} H^{i}(X, \mathbb{C})^{+} /\left(H^{i}(X, \mathrm{R}(n))^{+}+F^{n}\right), m<\frac{i}{2},
$$

follows from the shape of the $\Gamma$-factors and the conjectured functional equation, see [Be1, 3.3] or [J2, 4.14.a].

REMARK 3: The domain $m<0(\Leftrightarrow i+1<n)$ can be considered as the "stable range" for various reasons:

a) One has $F^{n} H^{i}(X, \mathbb{C})=0$ for the Hodge filtration.

b) One expects equality $K_{2 n-i-1}(X / \mathbb{Z})^{(n)}=K_{2 n-i-1+}(X)^{(n)}$ by conjectures on the $K$-theory of schemes over finite fields (cf. [Be1, 2.4.2]).

c) The local Euler factors are expected to be holomorphic here (see also the discussion in $§ 4$ ).

Via a) and b), Beilinson's conjecture predicts

$$
K_{2 n-i-1}(X)^{(n)} \otimes \mathrm{R} \stackrel{r}{\rightarrow} H^{i}(X, \mathbb{C})^{+} / H^{i}\left(X, \mathbb{R}(n)^{+} \text {for } m<0 .\right.
$$

Since $H^{i}(X, \mathbb{C})=H^{i}(X, \mathbf{R}) \otimes_{\mathbf{R}} \mathbb{C}$ is an induced module for $\langle\sigma\rangle$, we have $\operatorname{dim}_{\mathbf{R}} H^{i}(X, \mathbb{C})^{+}=\operatorname{dim}_{\mathbf{R}} H^{i}(X, \mathbf{R})$. Hence the dimension of the right-hand side of $(7)$ is $-\chi_{i, n}(X)$. On the other hand, c) would give

$$
\underset{s=m}{\operatorname{ord}} L(V, s)=\underset{s=m}{\operatorname{ord}} L_{S}(V, s) \text { for } m<0
$$

where $L_{S}(V, s)=\prod_{\mathfrak{p} \notin S} \operatorname{det}\left(1-\operatorname{Fr}_{\mathfrak{p}}(N \mathfrak{p})^{-s} \mid H^{i}\left(\bar{X}, Q_{\ell}\right)\right)^{-1}$ is the partial $L$-function.

We now consider an $\ell$-adic analogue of the above. Let $H_{\text {cont }}^{*}\left(X, \mathbb{Z}_{\ell}(n)\right)$ be the continuous étale cohomology of $X$ (see [DF] and [J1]). Then there is a Hochschild-Serre spectral sequence

$$
E_{2}^{p, q}=H^{p}\left(G_{k}, H^{q}\left(\bar{X}, \mathbb{Z}_{\ell}(n)\right)\right) \Rightarrow H_{\mathrm{cont}}^{p+q}\left(X, \mathbb{Z}_{\ell}(n)\right),
$$

see $[\mathbf{J} 1,3.4]$. There are also Chern characters on $K_{*}(X)$ with values in $H_{\text {cont }}^{*}\left(X, \mathbb{Q}_{\ell}(*)\right)=H_{\text {cont }}^{*}\left(X, \mathbb{Z}_{\ell}(*)\right) \otimes_{\mathbb{Z}_{\ell}} \mathbb{Q}_{\ell}$ (see [Sou3]), and, letting $H_{\text {cont }}^{\nu}\left(X, Q_{\ell}(n)\right)_{0}=\operatorname{Ker}\left(H_{\text {cont }}^{\nu}\left(X, Q_{\ell}(n)\right) \stackrel{\text { res }}{\rightarrow} H^{\nu}\left(\bar{X}, Q_{\ell}(n)\right)\right)$, (9) defines a 
diagram

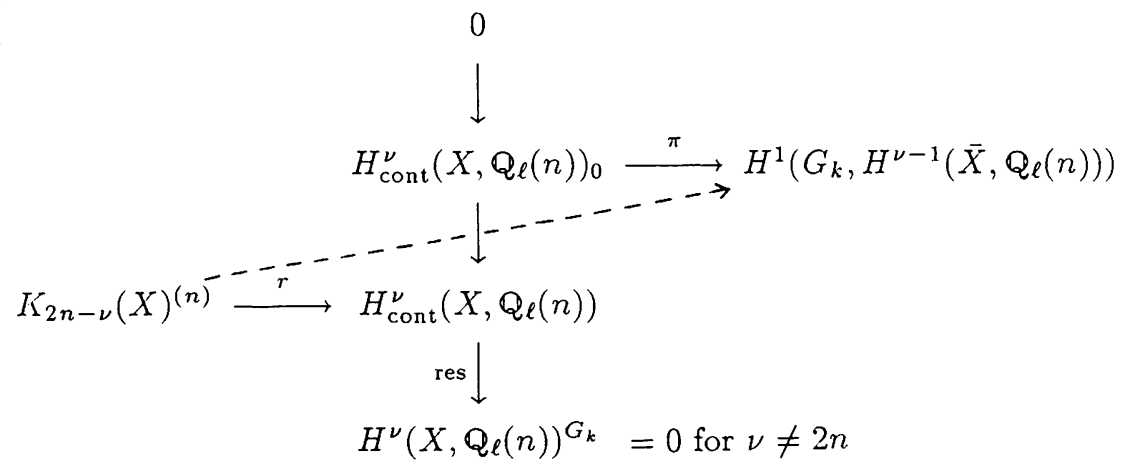

which should be considered as the $\ell$-adic analogue of (4). Since $c d_{\ell}\left(G_{k}\right) \leq 2$, $\pi$ is surjective. One can also show that res is surjective and that $\operatorname{Ker} \pi=$ $H^{2}\left(G_{k}, H^{\nu-2}\left(\bar{X}, Q_{\ell}(n)\right)\right)$, but this will not be needed in what follows.

CONJECTURE 2. The regulator map

$$
r: K_{2 n-i-1}(X)^{(n)} \otimes \mathbb{Q}_{\ell} \rightarrow H^{1}\left(G_{k}, H^{i}\left(\bar{X}, \mathbb{Q}_{\ell}(n)\right)\right)
$$

is an isomorphism for $i+1<n(\Longleftrightarrow m:=i+1-n<0)$.

The analogy with Beilinson's conjecture becomes quite clear by the formulae

$$
\begin{aligned}
H^{1}\left(G_{k}, H^{i}\left(\bar{X}, Q_{\ell}(n)\right)\right) & =\operatorname{Ext}_{G_{k}}^{1}\left(Q_{\ell}, H^{i}\left(\bar{X}, Q_{\ell}(n)\right)\right), \\
H^{i}(X, \mathbb{C})^{+} /\left(H^{i}(X, \mathbf{R}(n))^{+}+F^{n}\right) & =\operatorname{Ext}_{M H_{\mathbf{R}}}^{1}\left(\mathbf{R}, H^{i}(X, \mathbf{R}(n))\right),
\end{aligned}
$$

where $M H_{\mathbf{R}}$ is the category of real Hodge structures over $\mathbf{R}$ (i.e., with "infinite Frobenius $F_{\infty}$ "), see [J2, 4.13$]$. In fact, the philosophy behind both conjectures is that the motivic cohomology $H_{\mathcal{M}}^{i+1}=K_{2 n-i-1}(X)^{(n)}$ describes extensions of the trivial motive by the motive $H^{i}(X)(n)$, and that the regulator maps $r$ are obtained by passing to the associated realizations, see $[\mathrm{Be} 2,5,10],[\mathbf{D 5}]$, and $[\mathbf{J 2}, \S 4]$. In contrast to the Hodge realization, we have a group $H^{2}\left(G_{k}, H^{\nu-2}\left(\bar{X}, Q_{\ell}(n)\right)\right)=\operatorname{Ext}_{G_{k}}^{2}\left(\mathbf{Q}_{\ell}, H^{\nu-2}\left(\bar{X}, \mathbf{Q}_{\ell}(n)\right)\right)$ for the $\ell$-adic realizations, but comparison with Beilinson's conjecture and the general philosophy that there are "no non-trivial motivic 2-extensions over $\mathbf{Q}$ " up to torsion (see [D5]) suggests neglecting this group for the regulator map. 
All this is discussed in more detail in [J3], together with the function field analogue of Conjecture 2, which would follow from several well-known conjectures on $K$-theory and $\ell$-adic cohomology of varieties over finite fields. In these notes we restrict ourselves to the connection with Conjecture 1. We use the following fact, which has also been observed by P. Schneider and W. Raskind.

Lemma 4. If $i \neq 2 n-2$, then the inflation map

$$
H^{1}\left(G_{S}, H^{i}\left(\bar{X}, Q_{\ell}(n)\right)\right) \stackrel{\text { inf }}{\rightarrow} H^{1}\left(G_{k}, H^{i}\left(\bar{X}, Q_{\ell}(n)\right)\right)
$$

is an isomorphism and, consequently, $H^{1}\left(G_{k}, H^{i}\left(\bar{X}, Q_{\ell}(n)\right)\right)$ is finite-dimensional.

Proof: More generally, let $M$ be a finitely generated, torsion-free $\mathbb{Z}_{\ell^{-}}$ module with continuous action of $G_{S}$. Then there is an exact sequence

$$
0 \rightarrow H^{1}\left(G_{S}, M\right) \stackrel{\text { inf }}{\rightarrow} H^{1}\left(G_{k}, M\right) \rightarrow H^{1}\left(G_{k_{S}}, M\right)^{G_{S}}
$$

(by $[\mathbf{J} 1, \S 2]$, or by (1) and the well-known case of finite coefficients). By assumption, $G_{k_{S}}$ acts trivially on $M$. For every $\mathfrak{p} \notin S$ choose a decomposition group $G_{\mathfrak{p}} \subseteq G_{k}$ for $\mathfrak{p}$ and let $I_{\mathfrak{p}} \subset G_{\mathfrak{p}}$ be the inertia group. Then $G_{k_{S}}$ is the smallest closed normal subgroup of $G_{k}$ containing the $I_{\mathfrak{p}}, \mathfrak{p} \notin S$, and hence the restriction

$$
H^{1}\left(G_{k_{S}}, M\right)^{G_{S}}=\operatorname{Hom}_{\mathrm{cont}, G_{S}}\left(G_{k_{S}}^{\mathrm{ab}}, M\right) \hookrightarrow \prod_{\mathfrak{p} \notin S} \operatorname{Hom}_{\mathrm{cont}, G_{\mathrm{p}}}\left(I_{\mathfrak{p}}^{\mathrm{ab}}, M\right)
$$

is injective. Since $\mathfrak{p} \nmid \ell$ for $\mathfrak{p} \notin S$, there are isomorphisms

$$
\operatorname{Hom}_{\text {cont }, G_{\mathrm{p}}}\left(I_{\mathfrak{p}}^{\mathrm{ab}}, M\right) \cong \operatorname{Hom}_{\text {cont }, G_{\mathrm{p}}}\left(\mathbb{Z}_{\ell}(1), M\right) \cong M(-1)^{G_{\mathrm{p}}}
$$

by local class field theory. Hence the inflation is an isomorphism, if $M(-1)^{G_{p}}$ $=0$ for $\mathfrak{p} \notin S$. For $M=H^{1}\left(\bar{X}, \mathbb{Z}_{\ell}(n)\right) /$ torsion, this is the case for $i \neq 2(n-1)$ by Lemma 3 .

Corollary 2. If Beilinson's conjecture is true, then Conjecture 1 a) is equivalent to the equality of dimensions in Conjecture 2.

Proof: As explained in Remark 3, Beilinson's conjecture implies

$$
\operatorname{dim}_{Q} K_{2 n-i-1}(X)^{(n)}=-\chi_{i, n}(X) \text { for } i+1<n .
$$


Hence the claim is clear from Corollary 1 a) and Lemma 3.

Example 3 Consider $X=$ Spec $k$ : It follows from results of Borel and Soule that $K_{2 n}\left(\mathcal{O}_{k}\right)$ is finite for $n>0$ and that the $\ell$-adic Chern class

$$
c_{1, n}: K_{2 n-1}\left(\mathcal{O}_{k}\right) \otimes \mathbb{Z}_{\ell} \rightarrow H_{\text {ét }}^{1}\left(\operatorname{Spec}\left(\mathcal{O}_{k}\left[\frac{1}{\ell}\right]\right), \mathbb{Z}_{\ell}(n)\right)
$$

has finite kernel and cokernel for $n>1$, where $\mathcal{O}_{k}$ is the ring of integers in $k$ (see [Sou1] and [Sou2]). Now $H_{\text {êt }}^{1}\left(\operatorname{Spec}\left(\mathcal{O}_{k}\left[\frac{1}{\ell}\right]\right), \mathbb{Z}_{\ell}(n)\right)=H^{1}\left(G_{S}, \mathbb{Z}_{\ell}(n)\right)$ for $S=S_{\ell}$ (cf. (16) below), and $K_{r}\left(\mathcal{O}_{k}\right) \otimes \mathbb{Q} \rightarrow K_{r}(k) \otimes \mathbb{Q}$ for $r \geq 2$, as follows from the localization sequence and the finiteness of $K_{m}(F)$ for a finite field $F$ and $m \neq 0$. Finally, $c_{1, n}$ vanishes on $K_{2 n-1}\left(\mathcal{O}_{k}\right)^{(\nu)}$ for $\nu \neq n$ by standard properties of Chern classes. Putting all this together, one obtains the equality $K_{2 n-1}(k) \otimes \mathbb{Q}=K_{2 n-1}(k)^{(n)}$ for all $n \geq 1$ and the bijectivity of

$$
r: K_{2 n-1}(k)^{(n)} \otimes \mathbb{Q}_{\ell} \rightarrow H^{1}\left(G_{k}, \mathbb{Q}_{\ell}(n)\right), \quad n>1,
$$

using Lemma 4. Hence Conjecture 2 is true for $X=\operatorname{Spec} k$. Since, by Borel, Beilinson's conjectures are also true for $X=\operatorname{Spec} k$, we may deduce Conjecture 1 a) from this. The argument in [Sou1] is different: there it is proved that the Chern classes

$$
c_{2, n}: K_{2 n-2}\left(\mathcal{O}_{k}\right) \otimes \mathbb{Z}_{\ell} \rightarrow H_{\text {ét }}^{2}\left(\operatorname{Spec} \mathcal{O}_{k}\left[\frac{1}{\ell}\right], \mathbb{Z}_{\ell}(n)\right)
$$

have finite cokernels for $n>1$.

Example 4 Let $k$ be an imaginary quadratic field and let $X=E$ be an elliptic curve with complex multiplication by $k$. Assume that $\ell$ splits in $k$, $\ell=\mathfrak{p p}^{*}$, and that $E$ has good reduction at $\ell$. In [Sou5] Soule considers a map

$$
r^{\prime}: \lim _{\bar{r}} K_{2 n-2}\left(E, \mathbb{Z} / \ell^{r}\right) \rightarrow H^{1}\left(G_{k}, H^{1}\left(\bar{E}, \mathbb{Z}_{\ell}(n)\right)\right), \quad n>1 .
$$

Here $K_{2 n-2}\left(E, \mathbb{Z} / \ell^{r}\right)$ denotes $K$-theory with coefficients, sitting in an exact sequence

$$
0 \rightarrow K_{2 n-2}(E) / \ell^{r} \rightarrow K_{2 n-2}\left(E, \mathbb{Z} / \ell^{r}\right) \rightarrow K_{2 n-3}(E)_{\ell^{r}} \rightarrow 0
$$

and the composition of $r^{\prime}$ with $K_{2 n-2}(E) \rightarrow \lim _{\overleftarrow{r}} K_{2 n-2}\left(E, \mathbb{Z} / \ell^{r}\right)$ coincides with the $\ell$-adic Chern character up to some factorials after tensoring 
with $Q_{\ell}$. Soule finds that for a regular prime $\ell$ the composition of $r^{\prime}$ with the restriction

$$
\alpha_{\mathfrak{p}}: H^{1}\left(G_{k}, H^{1}\left(\bar{E}, \mathbb{Z}_{\ell}(n)\right)\right) \rightarrow H^{1}\left(G_{\mathfrak{p}}, H^{1}\left(\bar{E}, \mathbb{Z}_{\ell}(n)\right)\right), \quad n>1
$$

to a decomposition group $G_{\mathfrak{p}}$ at $\mathfrak{p}$ has a finite cokernel. On the other hand, it follows from the results of K. Wingberg mentioned in Example 2 that $\alpha_{\mathfrak{p}}$ has a finite kernel for regular $\ell$. Hence $r^{\prime}$ has a finite cokernel, in support of Conjecture 2.

In concordance with a conjecture of Quillen, Thomason [ $\mathrm{Th}$ ] has shown that for $\mu>N:=\frac{8}{3}(d+2)(d+3)(d+4)-15$ the map

$$
K_{\mu}\left(X, \mathbb{Z} / \ell^{r}\right) \rightarrow K_{\mu}^{\mathrm{e} t}\left(X, \mathbb{Z} / \ell^{r}\right)
$$

is a naturally split surjection, where $K_{\mu}^{\text {ét }}$ is the étale (or topological) $K$ theory. This implies that the Chern class

$$
c_{\nu, n}: \underset{\leftarrow}{\lim _{r}} K_{2 n-\nu}\left(X, \mathbb{Z} / \ell^{r}\right) \rightarrow \underset{\leftarrow}{\lim } H_{\text {ét }}^{\nu}\left(X, \mathbb{Z} / \ell^{r}(n)\right)
$$

has cokernel of finite exponent for $2 n>\nu+n$. On the other hand, the spectral sequence (9) induces a surjective map

$$
\underset{\leftarrow}{\lim } H_{\text {ét }}^{\nu}\left(X, \mathbb{Z} / \ell^{r}(n)\right)_{0} \stackrel{\pi}{\rightarrow} H^{1}\left(G_{k}, H^{\nu-1}\left(\bar{X}, \mathbb{Z}_{\ell}(n)\right)\right)
$$

where $H_{\text {ét }}^{\nu}\left(X, \mathbb{Z} / \ell^{r}(n)\right)_{0}=\operatorname{Ker}\left(H^{\nu}\left(X, \mathbb{Z} / \ell^{r}(n)\right) \stackrel{\text { res }}{\rightarrow} H^{\nu}\left(\bar{X}, \mathbb{Z} / \ell^{r}(n)\right)\right)$. In fact, we have seen that (9) gives a surjective map

$$
H_{\text {cont }}^{\nu}\left(X, \mathbb{Z}_{\ell}(n)\right)_{0} \stackrel{\pi}{\rightarrow} H^{1}\left(G_{k}, H^{\nu-1}\left(\bar{X}, \mathbb{Z}_{\ell}(n)\right)\right),
$$

and this map factors through $\lim _{\leftarrow} H_{\text {ét }}^{\nu}\left(X, \mathbb{Z} / \ell^{r}(n)\right)_{0}$, since $H^{1}\left(G_{k}\right.$, $\left.H^{\nu-1}\left(\bar{X}, \mathbb{Z}_{\ell}(n)\right)\right)=\lim _{\leftarrow} H^{1}\left(G_{k}, H^{\nu-1}\left(\bar{X}, \mathbb{Z} / \ell^{r}(n)\right)\right)$ is $\ell$-complete, see $[\mathbf{J} 1$, 4.4 and 5.16]. Altogether we obtain a surjective map

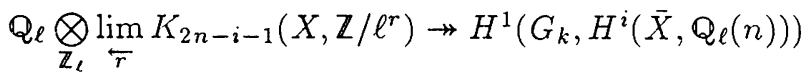

for $2 n>i+1+N$ (Note that $H^{\nu}(\bar{X}, \mathbb{Q}(n))^{G_{k}}=0$ for $\nu \neq 2 n$ ). By the exact sequences

$$
0 \rightarrow K_{\mu}(X)^{\wedge} \rightarrow \underset{\leftarrow}{\lim _{r}} K_{\mu}\left(X, \mathbb{Z} / \ell^{r}\right) \rightarrow T_{\ell} K_{\mu-1}(X) \rightarrow 0
$$


where $K_{\mu}(X)^{\wedge}=\lim _{\overleftarrow{r}} K_{\mu}(X) / \ell^{r}$ is the $\ell$-completion of $K_{\mu}(X)$, this would imply the surjectivity of the regulator map

$$
r: K_{2 n-i-1}(X)^{(n)} \otimes Q_{\ell} \rightarrow H^{1}\left(G_{k}, H^{i}\left(\bar{X}, Q_{\ell}(n)\right)\right)
$$

for $2 n>i+1+N$, if $T_{\ell} K_{2 n-i-2}(X)=0$, i.e., if $K_{2 n-i-2}(X)$ has no $\ell$ divisible torsion subgroup. Here we have used two facts: for an abelian group $A$, a finitely generated $\mathbb{Z}_{\ell}$-module $B$ and a homomorphism $f: A \rightarrow$ $B$, the induced map $A \otimes_{\mathbb{Z}} \mathbb{Q}_{\ell} \rightarrow B \otimes_{\mathbb{Z}_{\ell}} \mathbb{Q}_{\ell}$ is surjective if and only if the induced map $\hat{A} \otimes \mathbb{Z}_{\ell} \mathbb{Q}_{\ell} \rightarrow B \otimes \mathbb{Z}_{\ell} \mathbb{Q}_{\ell}$ is surjective (applied to $A=K_{2 n-i-1}(X)$ and $\left.B=H^{1}\left(G_{k}, H^{i}\left(\bar{X}, \mathbb{Z}_{\ell}(n)\right) / H^{i}\left(\bar{X}, \mathbb{Z}_{\ell}(n)\right)_{\text {tor }}\right)\right)$, and the Chern character $c h_{i+1, n}: K_{2 n-i-1}(X) \otimes \mathbb{Q} \rightarrow H^{1}\left(G_{k}, H^{i}\left(\bar{X}, Q_{\ell}(n)\right)\right)$ vanishes on $K_{2 n-i-1}(X)^{(r)}$ for $r \neq n$. Via the localization sequence

$$
\cdots \rightarrow K_{\mu}(\mathcal{X}) \rightarrow K_{\mu}(X) \rightarrow \bigoplus_{\mathfrak{p} \notin S} K_{\mu-1}\left(\mathcal{X}_{\mathfrak{p}}\right) \rightarrow K_{\mu-1}(\mathcal{X}) \rightarrow \ldots,
$$

where $\mathcal{X}$ is a smooth model of $X$ over the ring of $S$-integers $\mathcal{O}_{S}$ and $\mathcal{X}_{\mathfrak{p}}=$ $\mathcal{X} \times \mathcal{O}_{S} \kappa(\mathfrak{p})$ is the fiber at $\mathfrak{p}$, the vanishing of $T_{\ell} K_{\mu}(X)$ for all $\mu$ would follow from Bass's conjecture that $K_{\mu}(Y)$ is finitely generated for a regular scheme $Y$ of finite type over $\mathbb{Z}$.

In any case, if (12) is surjective, Beilinson's conjecture would also imply its injectivity. In fact, we would get $-\chi_{i, n}(X)=\operatorname{dim}_{\mathbb{Q}} K_{2 n-i-1}(X)^{(n)} \geq$ $\operatorname{dim}_{Q_{\ell}} H^{1}\left(G_{k}, H^{i}\left(\bar{X}, Q_{\ell}(n)\right)\right)$ and hence equality of dimensions by Corollary 1 a).

\section{Connections with Iwasawa theory.}

Let $M=H^{i}\left(\bar{X}, Q_{\ell} / \mathbb{Z}_{\ell}\right)$ and define

$$
\begin{array}{ll}
d_{i, n}=\operatorname{dim} H^{1}\left(G_{S}, M(n)\right), & d_{i, n}^{\prime}=\operatorname{dim} H^{1}\left(G_{k}, M(n)\right), \\
r_{i, n}=\operatorname{dim} H^{2}\left(G_{S}, M(n)\right), & r_{i, n}^{\prime}=\operatorname{dim} H^{2}\left(G_{k}, M(n)\right) .
\end{array}
$$

Lemma 5. For $i \neq 2 n-2$ these numbers are finite and independent of $S$, and one has $d_{i, n}=d_{i, n}^{\prime}, r_{i, n} \geq r_{i, n}^{\prime}$.

Proof: For any $\ell$-torsion $G_{S}$-module $N$ and any set of places $T \supseteq S$, one has an exact sequence

$$
\begin{aligned}
0 \rightarrow H^{1}\left(G_{S}, N\right) & \stackrel{\text { inf }}{\rightarrow} H^{1}\left(G_{T}, N\right) \rightarrow \bigoplus_{\mathfrak{p} \in T \backslash S} N(-1)^{G_{\mathrm{p}}} \\
& \rightarrow H^{2}\left(G_{S}, N\right) \stackrel{\text { inf }}{\rightarrow} H^{2}\left(G_{T}, N\right) \rightarrow \bigoplus_{\mathfrak{p} \in T \backslash S} N(-1)_{G_{\mathrm{p}}} \rightarrow 0,
\end{aligned}
$$


compare [Sou4, p. 117]. If $N$ is of cofinite type and if we assume

$$
N(-1)^{G_{\mathfrak{p}}} \text { and } N(-1)_{G_{\mathrm{p}}} \text { are finite for } \mathfrak{p} \in T \backslash S
$$

(when $N=H^{i}\left(\bar{X}, \mathbb{Q}_{\ell} / \mathbf{Z}_{\ell}(n)\right)$ this is the case for $i \neq 2(n-1)$ by the Wcil conjectures), then we immediately get

$$
\operatorname{dim} H^{\nu}\left(G_{S}, N\right)=\operatorname{dim} H^{\nu}\left(G_{T}, N\right), \quad \nu \geq 0, \quad \text { if } T \text { is finite. }
$$

If $T$ is the set of all places, then $G_{T}=G_{k}$, and we still get

$$
\begin{aligned}
& \operatorname{dim} H^{1}\left(G_{S}, N\right)=\operatorname{dim} H^{1}\left(G_{k}, N\right), \\
& \operatorname{dim} H^{2}\left(G_{S}, N\right) \geq \operatorname{dim} H^{2}\left(G_{k}, N\right),
\end{aligned}
$$

in view of the fact that $H^{2}\left(G_{S}, N\right)$ is cofinitely generated.

Lemma 6. Let $f_{S}: \mathcal{X}_{S} \rightarrow \operatorname{Spec} \mathcal{O}_{S}$ be a smooth and proper model of $X$ over $\mathcal{O}_{S}$, the ring of $S$-integers in $k$. If $\nu \neq 2 n-1,2 n, 2 n+1$, then

$$
\begin{aligned}
\operatorname{dim} H^{\nu}\left(\mathcal{X}_{S}, \mathbf{Q}_{\ell} / \mathbb{Z}_{\ell}(n)\right) & =d_{\nu-1, n}+r_{\nu-2, n} \\
& =-\chi_{\nu-1, n}+r_{\nu-1, n}+r_{\nu-2, n}, \\
\operatorname{dim} H^{\nu}\left(X, \mathbf{Q}_{\ell} / \mathbb{Z}_{\ell}(n)\right) & =d_{\nu-1, n}^{\prime}+r_{\nu-2, n}^{\prime} \\
& =-\chi_{\nu-1, n}+r_{\nu-1, n}+r_{\nu-2, n}^{\prime} .
\end{aligned}
$$

Proof: The first equality follows from the Leray spectral sequence

$$
E_{2}^{p, q}=H^{p}\left(\operatorname{Spec} \mathcal{O}_{S}, R^{q} f_{S_{*}} \mathbb{Q}_{\ell} / \mathbb{Z}_{\ell}(n)\right) \Rightarrow H^{p+q}\left(\mathcal{X}_{S}, Q_{\ell} / \mathbb{Z}_{\ell}(n)\right) .
$$

Namely, by base change one has an isomorphism of sheaves

$$
R^{q} f_{S} \mathbb{Q}_{\ell} / \mathbb{Z}_{\ell}(n) \cong j_{*} R^{q} f_{*} \mathbb{Q}_{\ell} / \mathbb{Z}_{\ell}(n)
$$

for the morphisms $f: X \rightarrow \operatorname{Spec} k$ and $j: \operatorname{Spec} k \hookrightarrow \operatorname{Spec} \mathcal{O}_{S}$, where $R^{q} f_{*} \mathbb{Q}_{\ell} / \mathbb{Z}_{\ell}(n)$ can be identified with the $G_{k}$-module $H^{q}\left(\bar{X}, \mathbb{Q}_{\ell} / \mathbb{Z}_{\ell}(n)\right)$. Since

$$
H^{p}\left(\operatorname{Spec} \mathcal{O}_{S}, j_{*} N\right)=H^{p}\left(G_{S}, N\right)
$$

for an $\ell$-torsion $G_{S}$-module $N$ (regarded as a sheaf on $\operatorname{Spec} k$ on the lefthand side), cf. [Mi2, II.2.9], we can rewrite (15) as

$$
E_{2}^{p, q}=H^{p}\left(G_{S}, H^{q}\left(\bar{X}, Q_{\ell} / \mathbb{Z}_{\ell}(n)\right) \Rightarrow H^{p+q}\left(\mathcal{X}_{S}, Q_{\ell} / \mathbb{Z}_{\ell}(n)\right) .\right.
$$


This implies the desired equality, since all groups occurring in (17) are cofinitely generated and $H^{q}\left(\bar{X}, \mathbb{Q}_{\ell} / \mathbb{Z}_{\ell}(n)\right)^{G_{k}}$ is finite for $q \neq 2 n$. For the latter reason, we have by Lemma 2

$$
-\chi_{i, n}=d_{i, n}-r_{i, n} \text { for } i \neq 2 n,
$$

which shows the second equality.

For the third equality, we use the Hochschild-Serre spectral sequence

$$
E_{2}^{p, q}=H^{p}\left(G_{k}, H^{q}\left(\bar{X}, \mathbf{Q}_{\ell} / \mathbb{Z}_{\ell}(n)\right) \Rightarrow H^{p+q}\left(X, \mathbf{Q}_{\ell} / \mathbb{Z}_{\ell}(n)\right) .\right.
$$

Since these groups in general are not cofinitely generated, we have to be a little bit careful with adding up dimensions in an exact sequence. Set $\left.H^{\nu}\left(X, \mathbb{Q}_{\ell} / \mathbb{Z}_{\ell}(n)\right)_{0}=\operatorname{Ker}\left(H^{\nu}\left(X, \mathbb{Q}_{\ell} / \mathbb{Z}_{\ell}(n)\right)\right) \stackrel{\text { res }}{\rightarrow} H^{\nu}\left(\bar{X}, \mathbb{Q}_{\ell} / \mathbb{Z}_{\ell}(n)\right)\right)$. Then (19) gives an exact sequence

$$
\begin{aligned}
H^{\nu-1}\left(\bar{X}, \mathbf{Q}_{\ell} / \mathbb{Z}_{\ell}(n)\right)^{G_{k}} & \rightarrow H^{2}\left(G_{k}, H^{\nu-2}\left(\bar{X}, \mathbf{Q}_{\ell} / \mathbb{Z}_{\ell}(n)\right)\right. \\
\rightarrow H^{\nu}\left(X, \mathbf{Q}_{\ell} / \mathbb{Z}_{\ell}(n)\right)_{0} & \rightarrow H^{1}\left(G_{k}, H^{\nu-1}\left(\bar{X}, \mathbf{Q}_{\ell} / \mathbb{Z}_{\ell}(n)\right) \rightarrow 0,\right.
\end{aligned}
$$

since $c d_{\ell}\left(G_{k}\right) \leq 2$. Now the second group in (20) is the direct sum of its maximal $\ell$-divisible subgroup and a group of finite exponent (since $H^{2}\left(G_{k}, N\right)$ is $\ell$-divisible for an $\ell$-divisible module $\left.N\right)$, and the first group is finite for $\nu-1 \neq 2 n$. From this we easily deduce

$$
\begin{aligned}
\operatorname{dim} & H^{2}\left(G_{k}, H^{\nu-2}\left(\bar{X}, \mathbb{Q}_{\ell} / \mathbb{Z}_{\ell}(n)\right)\right)+\operatorname{dim} H^{1}\left(G_{k}, H^{\nu-1}\left(\bar{X}, \mathbb{Q}_{\ell} / \mathbb{Z}_{\ell}(n)\right)\right) \\
& =\operatorname{dim} H^{\nu}\left(X, \mathbb{Q}_{\ell} / \mathbb{Z}_{\ell}(n)\right)_{0} \quad \text { for } \nu \neq 2 n+1 \\
& =\operatorname{dim} H^{\nu}\left(X, \mathbb{Q}_{\ell} / \mathbb{Z}_{\ell}(n)\right) \quad \text { if in addition } \nu \neq 2 n,
\end{aligned}
$$

and the last claimed equality again follows with (18).

Let $k_{\ell c}=k\left(\mu_{\ell \infty}\right)$ be the $\ell$-cyclotomic extension of $k$. Then $\operatorname{Gal}\left(k_{\ell c} / k\right) \cong$ $\Delta \times \Gamma$ with $\Gamma \cong \mathbb{Z}_{\ell}$ and $\# \Delta \mid(\ell-1)$. Let $H_{S}=\operatorname{Gal}\left(k_{S} / k_{\ell c}\right)$ and define

$$
\begin{aligned}
Y:=Y_{i}:=H^{1}\left(H_{S}, M\right)^{*}, & \tilde{Y}:=\tilde{Y}_{i}:=H^{1}\left(H_{S}, \tilde{M}\right)^{*}, \\
Z:=Z_{i}:=H^{1}\left(H_{S}, M\right)^{*}, & \tilde{Z}:=\tilde{Z}_{i}:=H^{1}\left(H_{S}, \tilde{M}\right)^{*},
\end{aligned}
$$

where $\tilde{M}=\ell-\operatorname{Div}(M)$ is the maximal divisible subgroup of $M$. These are compact $\Lambda$-modules, where $\Lambda=\mathbb{Z}_{\ell}[[\Gamma]]$ is the completed group ring. Let $\chi$ : $\operatorname{Gal}\left(k_{\ell c} / k\right) \rightarrow \mathbb{Z}_{\ell}^{\times}$be the $\ell$-cyclotomic character (defined by $\sigma(\zeta)=\zeta^{\chi(\sigma)}$ for $\zeta \in \mu_{\ell \infty}$ and $\left.\sigma \in \operatorname{Gal}\left(k_{\ell c} / k\right)\right)$ and let $e_{n}=(\# \Delta)^{-1} \sum_{\tau \in \Delta} \chi^{-n}(\tau) \tau \in \mathbb{Z}_{\ell}[\Delta]$ be the idempotent projecting to the part where $\Delta$ acts via $\chi^{n}(n \in \mathbb{Z})$. Of course, $e_{n}$ only depends on $n \bmod \# \Delta$. 
Lemma 7. a) For each $n \in \mathbf{Z}, Y e_{n}$ and $Z e_{n}$ are noetherian $\Lambda$-modules, and their ranks are independent of $S$.

b) $\operatorname{rank}_{\Lambda} Y e_{n}-\operatorname{rank}_{\Lambda} Z e_{n}=-\chi_{i, n}(X)$.

c) $\tilde{Z} e_{n}$ is a free $\Lambda$-module for all $n \in \mathbb{Z}$.

Proof: a) Let $H_{S}^{\prime}=\operatorname{Gal}\left(k_{S} / k_{\infty}\right)$, where $k_{\infty} / k$ is the unique $\mathbb{Z}_{\ell}$-extension contained in $k_{\ell c} / k$. Then we have canonical isomorphisms

$$
H^{r}\left(H_{S}^{\prime}, M(n)\right) \cong H^{r}\left(H_{S}, M(n)\right)^{\Delta} \cong\left(H^{r}\left(H_{S}, M\right) e_{-n}\right)(n), \quad r \geq 0,
$$

since $\ell \nmid \# \Delta$, so the dual of this module is noetherian if and only if $H^{r}\left(H_{S}, M\right)^{*} e_{n}$ is. Now the Hochschild-Serre spectral sequence for $1 \rightarrow$ $H_{S}^{\prime} \rightarrow G_{S} \rightarrow \Gamma \rightarrow 1$ gives exact sequences

$$
\begin{aligned}
& 0 \rightarrow H^{1}\left(\Gamma, M(n)^{H_{S}^{\prime}}\right) \rightarrow H^{1}\left(G_{S}, M(n)\right) \rightarrow H^{1}\left(H_{S}^{\prime}, M(n)\right)^{\Gamma} \rightarrow 0 \\
& 0 \rightarrow H^{1}\left(\Gamma, H^{1}\left(H_{S}^{\prime}(M(n))\right) \rightarrow H^{2}\left(G_{S}, M(n)\right) \rightarrow H^{2}\left(H_{S}^{\prime}, M(n)\right)^{\Gamma} \rightarrow 0\right.
\end{aligned}
$$

and an isomorphism

$$
H^{1}\left(\Gamma, H^{2}\left(H_{S}^{\prime}, M(n)\right)\right) \cong H^{3}\left(G_{S}, M(n)\right)=0,
$$

since $c d_{\ell} \Gamma=1, c d_{\ell} G_{S} \leq 2$ and $c d_{\ell} H_{S}^{\prime} \leq 2$. Since $H^{r}\left(G_{S}, M(n)\right)$ is cofinitely generated for all $r$ and $n$, the same is true for $H^{r}\left(H_{S}^{\prime}, M(n)\right)^{\Gamma}$. Hence $\left(H^{r}\left(H_{S}^{\prime}, M(n)\right)^{*}\right)_{\Gamma}$ is finitely generated over $\mathbb{Z}_{\ell}$. This implies that $H^{r}\left(H_{S}^{\prime}, M(n)\right)^{*}$ is finitely generated over $\Lambda$. For the second claim let $T$ be a finite set of places containing $S$. By applying (13) to each intermediate layer $k \subseteq k_{r} \subseteq k_{\infty}$ and passing to the limit over $r$, we get an exact sequence

$$
\begin{aligned}
0 \rightarrow H^{1}\left(H_{S}^{\prime}, M(n)\right) \rightarrow & H^{1}\left(H_{T}^{\prime}, M(n)\right) \rightarrow \bigoplus_{\mathfrak{p} \in T \backslash S\left(k_{\infty}\right)} M(n-1) \\
& \rightarrow H^{2}\left(H_{S}^{\prime}, M(n)\right) \rightarrow H^{2}\left(H_{T}^{\prime}, M(n)\right) \rightarrow 0,
\end{aligned}
$$

since $\lim _{\overleftarrow{r}} M(n-1)_{\mathrm{Gal}\left(k_{\infty} / k_{r}\right)}=0$ (the limit is taken via norms). The set $T \backslash S\left(k_{\infty}\right)$ of places in $k_{\infty}$ above $T \backslash S$ is finite, since all primes $\mathfrak{p}$ decompose into only finitely many primes in the cyclotomic extension. Therefore $\left(\bigoplus_{M} M(n-1)\right)^{*}$ is a $\Lambda$-torsion module (in fact, finitely generated $p \in T \backslash S\left(k_{\infty}\right)$

over $\mathbb{Z}_{\ell}$ ), and the claim follows, in view of (21).

For b), we use the fact that

$$
\operatorname{rank}_{\Lambda} W=\operatorname{rank}_{\mathbb{Z}_{\ell}} W_{\Gamma}-\operatorname{rank}_{\mathbb{Z}_{\ell}} W^{\Gamma}
$$


for a noetherian $\Lambda$-module $W$ (see, e.g., [Wi1 (1.4)]). Hence by (22) and (23) we get

$\operatorname{rank}_{\Lambda} Y e_{n}-\operatorname{rank}_{\Lambda} Z e_{n}=\operatorname{rank}_{\Lambda} H^{1}\left(H_{S}^{\prime}, M(n)\right)^{*}-\operatorname{rank}_{\Lambda} H^{2}\left(H_{S}^{\prime}, M(n)\right)^{*}$ $=\operatorname{dim} H^{1}\left(G_{S}, M(n)\right)-\operatorname{dim} H^{2}\left(G_{S}, M(n)\right)-\operatorname{dim} H^{1}\left(\Gamma, M(n)^{H_{S}^{\prime}}\right)=-\chi_{i, n}$, since $\operatorname{dim} H^{1}\left(\Gamma, M(n)^{H_{S}^{\prime}}\right)=\operatorname{dim} M(n)^{G_{s}}$ by the exact sequence

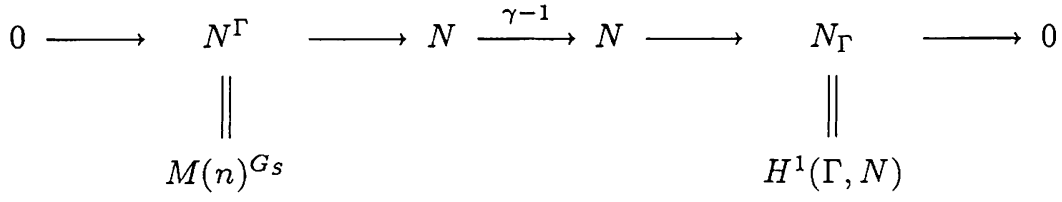

for $N=M(n)^{H_{S}^{\prime}}$ and $\gamma$ a topological generator of $\Gamma$.

For c), we use the criterion (see [Wi1 (1.2)])

$W$ free $\Longleftrightarrow W^{\Gamma}=0$ and $W_{\Gamma}$ is torsion-free

for the $\Lambda$-module $W=H^{2}\left(H_{S}^{\prime}, \tilde{M}(n)\right)^{*}$. Namely, $H^{2}\left(H_{S}^{\prime}, \tilde{M}(n)\right)_{\Gamma} \cong$ $H^{1}\left(\Gamma, H^{2}\left(H_{S}^{\prime}, \tilde{M}(n)\right)\right)=0$ by $(24)$, and $H^{2}\left(H_{S}^{\prime}, \tilde{M}(n)\right)^{\Gamma}$ is $\ell$-divisible as quotient of the $\ell$-divisible group $H^{2}\left(G_{S}, \tilde{M}(n)\right)$.

Iwasawa's conjecture " $\mu=0$ " for all number fields $K$ with $k \subseteq K \subseteq k_{S}$ would imply $c d_{\ell}\left(H_{S}\right)=1$ (see [Sch3, 3.8]) and in particular the vanishing of $Z=H^{2}\left(H_{S}, M\right)^{*}$. In any case, we have

LEMma 8. Let $G_{S, r}=\operatorname{Gal}\left(k_{S} / k_{r}\right)$, where $k_{r}=k\left(\mu_{\ell^{r}}\right) \cap k_{\infty}$. Then the following statement are equivalent for $n \in \mathbf{Z}$ :

a) $Z e_{n}$ is a $\Lambda$-torsion module.

b) $H^{2}\left(H_{S}, \tilde{M}\right) e_{-n}=0$.

c) $\operatorname{dim} H^{2}\left(G_{S, r}, M(n)\right)$ is bounded for $r \geq 1$.

c) For all $n^{\prime} \in \mathbf{Z}$ with $n^{\prime} \equiv n \bmod \# \Delta, \operatorname{dim} H^{2}\left(G_{S, r}, M\left(n^{\prime}\right)\right)$ is bounded for $r \geq 1$.

d) $H^{2}\left(G_{S}, M(n)\right)$ is finite for one $n^{\prime} \in \mathbb{Z}$ with $n^{\prime} \equiv n \bmod \# \Delta$.

$\left.\mathrm{d}^{\prime}\right) H^{2}\left(G_{S}, M\left(n^{\prime}\right)\right)$ is finite for almost all $n^{\prime} \in \mathbb{Z}$ with $n^{\prime} \equiv n \bmod \# \Delta$.

Proof: Since $\operatorname{rank}_{\Lambda} Z e_{n}=\operatorname{rank}_{\Lambda} \tilde{Z} e_{n}$, and $\tilde{Z} e_{n} \cong H^{2}\left(H_{S}^{\prime}, \tilde{M}(n)\right)^{*}(n)$ is $\Lambda$-free, the equivalence of a) and b) is clear. The equivalence with c) and d) follows from Lemma 1 and the exact sequences

$$
\begin{aligned}
0 \rightarrow H^{1}\left(\Gamma_{r}, H^{1}\left(H_{S}^{\prime}, \tilde{M}\left(n^{\prime}\right)\right)\right) \rightarrow H^{2}\left(G_{S, r}, \tilde{M}\left(n^{\prime}\right)\right) & \\
& \rightarrow H^{2}\left(H_{S}^{\prime}, \tilde{M}\left(n^{\prime}\right)\right)^{\Gamma_{r}} \rightarrow 0,
\end{aligned}
$$


$\Gamma_{r}=\operatorname{Gal}\left(k_{\infty} / k_{r}\right)$. Indeed, $\operatorname{dim}\left(\Lambda^{*}\right)^{\Gamma_{r}}=\left[k_{r}: k\right]$, and for a noetherian $\Lambda$-module $W$ the numbers

$$
\operatorname{dim} H^{1}\left(\Gamma_{r}, W^{*}(\nu)\right)
$$

are bounded for $r \geq 0, \nu \in \mathbb{Z}$, and zero for almost all twists $\nu \in \mathbb{Z}$, cf. [Sou4, Lemma 2]. Note that $H^{r}\left(H_{S}^{\prime}, \tilde{M}\left(n^{\prime}\right)\right)(\nu)=H^{r}\left(H_{S}^{\prime}, \tilde{M}\left(n^{\prime}+\nu\right)\right)$ for $\nu \equiv 0 \bmod \# \Delta$. The equivalence with $\left.c^{\prime}\right)$ and $\left.\mathrm{d}^{\prime}\right)$ is now clear, since a) and b) only depend on $n \bmod \# \Delta$.

In general, we obtain from (23)

$$
\begin{aligned}
r_{i, n} & =\operatorname{dim} H^{2}\left(G_{S}, M(n)\right)=\operatorname{rank}_{\Lambda} Z_{i} e_{n}+\operatorname{rank}_{\mathbb{Z}_{\ell}}\left(Y_{i} e_{n}(-n)\right)^{\Gamma} \\
& =\operatorname{rank}_{\Lambda} Z_{i} e_{n} \text { for almost all } n \in \mathbb{Z},
\end{aligned}
$$

and, via Lemma 6 and Corollary 4 below,

$$
\operatorname{dim} H^{\nu}\left(X, \mathbb{Q}_{\ell} / \mathbb{Z}_{\ell}(n)\right)=-\chi_{\nu-1, n}+\operatorname{rank}_{\Lambda} Z_{\nu-1} e_{n}
$$

for almost all $n \in \mathbb{Z}$.

These are the correct versions of Proposition 3 and Theorem 1 ii) in [Sou4], as long as we do not know the vanishing of $\tilde{Z}$. The gap in loc. cit. 1.5 is that one cannot conclude $c d_{p} H=1$ from $c d_{p} H_{p}=1$ without any extra argument, but the reasoning there still gives the following interesting result:

Lemma 9. Let $K$ be a finite extension of $k$ over which $\tilde{M}(n)_{\ell}$ becomes a trivial Galois module, let $K_{\infty}=K \cdot k_{\infty}$ be the cyclotomic $\mathbb{Z}_{\ell}$-extension of $K$, and let $\mathcal{M}$ be the maximal abelian $\ell$-extension of $K_{\infty}$ which is unramified outside $\ell$. If the $\mu$-invariant of the noetherian $\mathbb{Z}_{\ell}\left[\left[\mathrm{Gal}\left(K_{\infty} / K\right)\right]\right]$-module $\operatorname{Gal}\left(\mathcal{M} / K_{\infty}\right)$ is zero, then $H^{2}\left(H_{S}, \tilde{M}\right) e_{-n}=0$.

Proof: It follows from the assumption on the $\mu$-invariant that $H_{S}^{\prime}(K)(\ell)$, the maximal pro- $\ell$-factor group of $H_{S}^{\prime}(K)=\operatorname{Gal}\left(k_{S} / K_{\infty}\right)$, is pro- $\ell$-free (see [Wi1, 5.4 and 5.6]). Now by definition $G_{K}$ lies in the kernel of the composite of the two homomorphisms

$$
G_{k} \stackrel{\rho}{\rightarrow} \operatorname{Aut}(\tilde{M}(n)) \stackrel{\pi}{\rightarrow} \operatorname{Aut}\left(\tilde{M}(n)_{\ell}\right)
$$

given by the action of $G_{k}$ on $\tilde{M}(n)$ and the restriction to $\tilde{M}(n)_{\ell}$. Since Ker $\pi$ is a pro- $\ell$-group, $G_{K}$ and consequently $H_{S}^{\prime}(K)$ act on $\tilde{M}(n)$ via their pro- $\ell$-quotients. Hence we can consider the inflation

$$
H^{2}\left(H_{S}^{\prime}(K)(\ell), \tilde{M}(n)\right) \stackrel{\text { inf }}{\rightarrow} H^{2}\left(H_{S}^{\prime}(K), \tilde{M}(n)\right)
$$


and this map is an isomorphism (see [Neu, Cor. 1]). Furthermore the corestriction

$$
H^{2}\left(H_{S}^{\prime}(K), \tilde{M}(n)\right) \stackrel{\text { cor }}{\longrightarrow} H^{2}\left(H_{S}^{\prime}, \tilde{M}(n)\right)
$$

is surjective, since $c d_{\ell}\left(H_{S}^{\prime}\right) \leq 2$ (see [Se1, I 3.3]). It follows that $c d_{\ell}\left(H_{S}^{\prime}(K)(\ell)\right) \leq 1$ implies $H^{2}\left(H_{S}^{\prime}, \tilde{M}(n)\right)=0$, hence the result, by $(21)$.

The conjectured vanishing of $\tilde{Z}$, which would follow both from Iwasawa's " $\mu=0$ " conjecture or from Conjecture 1, may be more easily accessible than either of these. For example, in the case $X=\operatorname{Spec} k$ one can in fact prove $H^{2}\left(H_{S}, \mathbf{Q}_{\ell} / \mathbb{Z}_{\ell}\right)=0$ (which is also called the weak Leopoldt conjecture) without proving anything about $c d_{\ell} H_{S}$ or Conjecture 1 , see [Sch1, 4.7].

\section{Global results.}

Conjecture 1 is in part motivated by the following result.

THEOREM 1. The analogue of Conjecture 1 is true in the function field case.

This follows from the following, more general, fact.

THEOREM 2. Let $U$ be a smooth curve over a finite field $F_{q}$ and let $F$ be

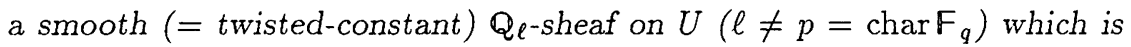
mixed $[\mathbf{D} 4,1.2 .2]$. Then $H^{2}(U, F)=0$ if

a) $F^{\vee}(2)$ is entire $[\mathrm{D} 4,3.3 .2]$, or

b) the weights in $F$ are $\geq 0$.

(Here $F^{\vee}$ is the dual of $F$, and $F^{\vee}(n)$ is its $n$-fold Tate twist.)

Corollary 3. Let $F$ be smooth of weight $i$ (see [D4, 1.2.2]: for each closed point $x \in U$ the eigenvalues $\alpha$ of the geometric Frobenius $\operatorname{Fr}_{x}$ on the stalk $F_{\bar{x}}$ are algebraic numbers with absolute value $|\alpha|=(N x)^{i / 2}$ for every complex embedding). Assume that $F$ is positive (or effective), by which we mean the following: $F$ is entire (i.e., the $\alpha$ are algebraic integers), and $F^{\vee}(-i)$ is entire. Then $H^{2}(U, F(n))=0$ if
a) $i+1<n$, or
b) $i+1>2 n$. 
Proof of Corollary 3: If $F^{\vee}(-i)$ is entire, then $F(n)^{\vee}(2)=F^{\vee}(-i)(i+$ $2-n)$ is entire for $i+2-n \leq 0$, since $Q_{\ell}(\nu)$ is entire for $\nu \leq 0$. If the weight of $F$ is $i$, then the weight of $F(n)$ is $i-2 n$, which is $\geq 0$ for $i+1>2 n$.

CoROllary $3 \Rightarrow$ THEOREM 1: Let $X$ be a smooth, proper variety over the function field $k=\mathrm{F}_{q}(U)$ of $U$ and assume that $X$ has a smooth, proper model $f: \mathcal{X} \rightarrow U$ (i.e., good reduction over $U$ ). By smooth and proper base change, $F=R^{i} f_{*} \mathrm{Q}_{\ell}$ is a twisted-constant sheaf on $U$, and for each $x \in U$ there is an isomorphism $F_{\bar{x}} \cong H^{i}\left(\overline{\mathcal{X}}_{x}, Q_{\ell}\right)$, where $\mathcal{X}_{x}=\mathcal{X} \times_{U} \operatorname{Spec} \kappa(x)$ is the fiber of $f$ at $x$ and $\overline{\mathcal{X}}_{x}=\mathcal{X}_{x} \times_{\kappa(x)} \overline{\kappa(x)}$ for a separable closure $\overline{\kappa(x)}$ of the residue field $\kappa(x)$ of $x$. If $U$ is affine, and $\eta=\operatorname{Spec} k$ is the generic point of $U$, then there are canonical isomorphisms

$$
H^{r}(U, F(n)) \cong H^{r}\left(\pi_{1}(U, \bar{\eta}), F(n)_{\bar{\eta}}\right) \cong H^{r}\left(\pi_{1}(U, \bar{\eta}), H^{i}\left(\bar{X}, Q_{\ell}(n)\right)\right),
$$

for all $r \geq 0$, where $\bar{\eta}$ is the geometric point over $\eta$ given by Spec $\bar{k}$, see [Mi2, II 2.9]. In this situation, $U$ is the analogue of $\operatorname{Spec} \mathcal{O}_{S}$; note that by definition $G_{S}=\pi_{1}\left(\operatorname{Spec} \mathcal{O}_{S}, \bar{\eta}\right)$ in the number field situation.

To apply Corollary 3 , we have to show that $F$ is positive. But by Deligne's proof of the Weil conjectures, $H^{i}\left(\overline{\mathcal{X}}_{x}, Q_{\ell}\right)$ is entire of weight $i$ for $x \in U$ closed, and by Poincaré duality and hard Lefschetz [D4, 4.1.1]

$$
H^{i}\left(\overline{\mathcal{X}}_{x}, \mathbf{Q}_{\ell}\right)^{\vee}(-i) \cong H^{2 d-i}\left(\overline{\mathcal{X}}_{x}, \mathbf{Q}_{\ell}\right)(d-i) \cong H^{i}\left(\overline{\mathcal{X}}_{x}, \mathbf{Q}_{\ell}\right)
$$

is again entire $(d=\operatorname{dim} X)$.

Proof of ThEOREM 2: If the weights in $F$ are $\geq r$, then the weights in $F^{\vee}$ are $\leq-r$. By Deligne's fundamental result [D4, 3.3.4], the weights $w^{\prime}$ occurring in $H_{c}^{1}\left(\bar{U}, F^{\vee}(1)\right)$, the cohomology with compact support of $\bar{U}=$ $U \times \mathbf{F}_{q} \overline{\mathbf{F}}$, satisfy $w^{\prime} \leq-r+1-2=-r-1$ (note that $Q_{\ell}(1)$ has weight -2 ), and if $F^{\vee}(2)$ is entire, we have $w^{\prime}-2 \geq 0$, see [D4, 3.3.3]. By Poincaré duality, we have

$$
H^{1}(\bar{U}, F) \cong H_{c}^{1}\left(\bar{U}, F^{\vee}(1)\right)^{\vee} .
$$

Hence for the weights $w$ occurring in this mixed $G_{\mathbf{F}_{q}}$-module, where $G_{\mathbf{F}_{q}}=$ $\operatorname{Gal}\left(\overline{\mathbf{F}}_{q} / \mathbf{F}_{q}\right)$, we have $w \geq r+1$, and $w \leq-2$ under assumption a). Similarly, $H^{2}(\bar{U}, F) \cong H_{c}^{0}\left(\bar{U}, F^{\vee}(1)\right)^{\vee}$ has weights $\geq r+2$, and $\leq-2$ under assumption a). We apply this to the exact sequence

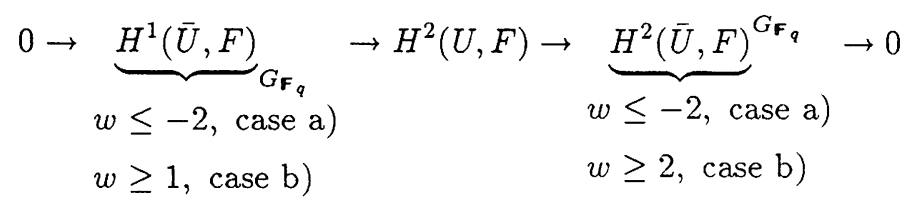


obtained from the Hochschild-Serre spectral sequence for $\overline{\mathbf{F}}_{q} / \mathrm{F}_{q}$. We conclude that $H^{2}(U, F)=0$ in both cases, since a mixed $G_{\mathbf{F}_{q}}$-module can have a non-zero fixed or cofixed module only if the weight zero occurs.

REMARK 4: The bounds in Theorem 2 seem to be sharp, and I expect the same of the bounds in Conjecture 1, except for an improvement by hard Lefschetz: $H^{i}\left(\bar{X}, Q_{\ell}\right) \cong H^{2 d-i}\left(\bar{X}, Q_{\ell}(d-i)\right)$, which would imply $H^{2}\left(G_{S}, H^{i}\left(\bar{X}, Q_{\ell}(n)\right)\right)=0$ for $n>d+1$.

Question 1 Is the analogue of Theorem 2 true in the number field case ( $U$ being replaced by $\operatorname{Spec} \mathcal{O}_{S}$ for a finite set $S$ of primes containing all primes above $\ell)$ ?

By the same arguments as above, a positive answer to this question would imply Conjecture 1. So far, one does not know any number field analogue of Deligne's theorem that $H_{c}^{\nu}(\bar{U}, F)$ is mixed of weights $\leq \nu+r$, if $F$ is mixed of weights $\leq r$, or, what is more or less the same, the analogous result for $H^{\nu}(\bar{Y}, G)$, where $Y$ is the smooth compactification of $U$. According to the general philosophy of Iwasawa theory, the analogue of $H^{\nu}(\bar{Y}, G)$ should be a certain Iwasawa module over $k_{\infty}$ - for example, the Galois group $\operatorname{Gal}\left(L / k_{\infty}\right)$ of the maximal abelian unramified $\ell$-extension $L$ of $k_{\infty}$ for $\nu=1$ and $G=Q_{\ell}(1)$. But no integrality results exist in this setting and perhaps cannot be expected in a "naive" way, cf. [Wa2]. Is it possible that, nevertheless, there exists some theory of weights for these Iwasawa modules?

We return to number fields and to the notations at the beginning of $\S 1$. It turns out that the numbers $r_{i, n}^{\prime}=\operatorname{dim} H^{2}\left(G_{k}, H^{i}\left(\vec{X}, Q_{\ell} / \mathbb{Z}_{\ell}(n)\right)\right)$ are much more accessible than the numbers $r_{i, n}=\operatorname{dim} H^{2}\left(G_{S}, H^{i}\left(\bar{X}, Q_{\ell} / \mathbb{Z}_{\ell}(n)\right)\right)$.

TheOREM 3. Let $P$ be a set of primes of $k$ of density 1 .

a) The localization map (induced by the restrictions to the decomposition groups $G_{\mathfrak{p}}$ )

$$
\alpha_{1}^{i, n}: H^{1}\left(G_{k}, H^{i}\left(\bar{X}, \mathbb{Z}_{\ell}(n)\right)\right) \rightarrow \prod_{\mathfrak{p} \in P} H^{1}\left(G_{\mathfrak{p}}, H^{i}\left(\bar{X}, \mathbb{Z}_{\ell}(n)\right)\right)
$$

has a finite kernel for $i \neq 2 n$.

b) Let $\tilde{H}^{i}\left(\bar{X}, \mathbb{Z}_{\ell}(n)\right)=H^{i}\left(\bar{X}, \mathbb{Z}_{\ell}(n)\right) / H^{i}\left(\bar{X}, \mathbb{Z}_{\ell}(n)\right)_{\text {tors }}$, where $A_{\text {tors }}$ is the torsion subgroup of an abelian group $A$. Then

$$
\tilde{\alpha}_{1}^{i, n}: H^{1}\left(G_{k}, \tilde{H}^{i}\left(\bar{X}, \mathbb{Z}_{\ell}(n)\right)\right) \rightarrow \prod_{\mathfrak{p} \in P} H^{1}\left(G_{\mathfrak{p}}, \tilde{H}^{i}\left(\bar{X}, \mathbb{Z}_{\ell}(n)\right)\right)
$$


is injective for $i \neq 2 n$.

c) The localization map

$$
\alpha_{2}^{i, n}: H^{2}\left(G_{k}, H^{i}\left(\bar{X}, \mathbb{Q}_{\ell} / \mathbb{Z}_{\ell}(n)\right)\right) \rightarrow \bigoplus_{\text {all } \mathfrak{p}} H^{2}\left(G_{\mathfrak{p}}, H^{i}\left(\bar{X}, \mathbb{Q}_{\ell} / \mathbb{Z}_{\ell}(n)\right)\right)
$$

has a finite kernel and cokernel for $i \neq 2(n-1)$.

d) Let $\tilde{H}^{i}\left(\bar{X}, \mathbb{Q}_{\ell} / \mathbb{Z}_{\ell}(n)\right)=\ell-\operatorname{Div} H^{i}\left(\bar{X}, \mathbb{Q}_{\ell} / \mathbb{Z}_{\ell}(n)\right)$. Then

$$
\tilde{\alpha}_{2}^{i, n}: H^{2}\left(G_{k}, \tilde{H}^{i}\left(\bar{X}, Q_{\ell} / \mathbb{Z}_{\ell}(n)\right)\right) \rightarrow \bigoplus_{\mathfrak{p} \in S} H^{2}\left(G_{\mathfrak{p}}, H^{i}\left(\bar{X}, \mathbb{Q}_{\ell} / \mathbb{Z}_{\ell}(n)\right)\right)
$$

is an isomorphism for $i \neq 2(n-1)$.

Proof: This follows directly from results of Serre: For a profinite group $G$ and a topological $G$-module $M$ let

$$
H_{*}^{1}(G, M)=\operatorname{Ker}\left(H^{1}(G, M) \stackrel{\text { res }}{\rightarrow} \prod_{C} H^{1}(C, M)\right),
$$

where $C$ runs over all (pro-) cyclic subgroups of $G$ (and the cohomology is the continuous one, as always). If $\mathcal{G}=\operatorname{Im}(G \rightarrow \operatorname{Aut}(M))$, then

$$
H_{*}^{1}(\mathcal{G}, M) \stackrel{\inf }{\rightarrow} H_{*}^{1}(G, M)
$$

[Se2, Prop. 6]. We now consider $M=H^{i}\left(\bar{X}, \mathbb{Z}_{\ell}(n)\right), i \neq 2 n$, and $G=G_{k}$. Then, by the Weil conjectures proved by Deligne, for a Frobenius $\operatorname{Fr}_{\mathfrak{p}} \in \mathcal{G}$ at $\mathfrak{p} \notin S$ its eigenvalues on $M$ are of weight $i-2 n \neq 0$ and thus satisfy the condition in Lemma 2 of [Se3]. Hence the Lie algebra $\mathfrak{g}$ of $\mathcal{G}$ satisfies the hypothesis of Theorem 1 in [Se3], and we get

$$
H^{r}\left(\mathfrak{g}, M \otimes \mathbb{Q}_{p}\right)=0 \text { for all } r \geq 0
$$

Hence

$$
H^{r}(\mathcal{G}, M) \text { is finite for all } r \geq 0
$$

by results of Lazard, which show that $H^{r}(\mathcal{G}, M)$ is a finitely generated $\mathbb{Z}_{\ell^{-}}$ module for all $r$ and that there is an injection $H^{r}(\mathcal{G}, M) \otimes Q_{\ell} \hookrightarrow H^{r}(\mathfrak{g}, M \otimes$ $\left.Q_{\ell}\right)$, cf. [Se3, Cor. to Lemma 3]. Moreover, since $\left(M \otimes Q_{p}\right)^{F_{r_{p}}}=0$, we have

$$
H_{*}^{1}\left(\mathcal{G}, \tilde{H}^{i}\left(\bar{X}, \mathbb{Z}_{\ell}(n)\right)\right)=0 \quad(i \neq 2 n),
$$


see [Se2, Theorem 1]. This implies b), since the kernel of the map in b) is contained in $H_{*}^{1}\left(\mathcal{G}, \tilde{H}^{i}\left(\bar{X}, \mathbb{Z}_{\ell}(n)\right)\right)$ ([Se2, Prop. 7 and 8$]$ - the proof also works for a set of primes of density 1 , cf. [Se4, II Notes].

For a) we may assume without restriction that $S \cap P=\emptyset$. Then $\tilde{H}^{i}\left(\bar{X}, \mathbb{Z}_{\ell}(n)\right)^{G_{\mathfrak{p}}}=0$ for all $\mathfrak{p} \in P(i \neq 2 n)$. Thus the claim easily follows via the exact sequence

$$
0 \rightarrow H^{i}\left(\bar{X}, \mathbb{Z}_{\ell}(n)\right)_{\text {tors }} \rightarrow H^{i}\left(\bar{X}, \mathbb{Z}_{\ell}(n)\right) \rightarrow \tilde{H}^{i}\left(\bar{X}, \mathbb{Z}_{\ell}(n)\right) \rightarrow 0,
$$

and the fact that the localization map for the finite module $H^{i}\left(\bar{X}, \mathbb{Z}_{\ell}(n)\right)_{\text {tors }}$ has a finite kernel by the theorem of Tate and Poitou [Ta1, 3.1].

When $P$ is the set of all primes, the cited global duality theorem also gives an exact sequence

$$
\begin{aligned}
& 0 \rightarrow\left(\operatorname{Ker} \alpha_{1}^{2 d-i, d+1-n}\right)^{*} \rightarrow H^{2}\left(G_{k}, H^{i}\left(\bar{X}, Q_{\ell} / \mathbb{Z}_{\ell}(n)\right)\right) \\
& \stackrel{\alpha_{2}^{i, n}}{\longrightarrow} \underset{\text { all } \mathfrak{p}}{\bigoplus_{1}} H^{2}\left(G_{\mathfrak{p}}, H^{i}\left(\bar{X}, \mathbb{Q}_{\ell} / \mathbb{Z}_{\ell}(n)\right)\right) \rightarrow H^{i}\left(\bar{X}, \mathbf{Q}_{\ell} / \mathbb{Z}_{\ell}(n-1)\right)_{G_{k}} \rightarrow 0,
\end{aligned}
$$

by passing to the limit over the corresponding exact sequences for $H^{i}\left(\bar{X}, \mathbf{Z} / \ell^{r}(n)\right)$. Here we have used the fact that

$$
\operatorname{Hom}\left(H^{i}\left(\bar{X}, \mathbf{Q}_{\ell} / \mathbb{Z}_{\ell}(n)\right), \mathbf{Q}_{\ell} / \mathbb{Z}_{\ell}(1)\right) \cong H^{2 d-i}\left(\bar{X}, \mathbb{Z}_{\ell}(d+1-n)\right)
$$

by Poincaré duality, and that

$$
H^{0}\left(G_{k}, \operatorname{Hom}\left(A, Q_{\ell} / \mathbb{Z}_{\ell}(1)\right)\right)^{*} \cong \operatorname{Hom}_{G_{k}}\left(A(-1), \mathbb{Q}_{\ell} / \mathbb{Z}_{\ell}\right)^{*} \cong A(-1)_{G_{k}}
$$

for the $\ell$-torsion $G_{k}$-module $A=H^{i}\left(\bar{X}, Q_{\ell} / \mathbb{Z}_{\ell}(n)\right)$. Now $\operatorname{Ker} \alpha_{1}^{2 d-i, d+1-n}$ is finite for $i \neq 2(n-1)$ by a), and $H^{i}\left(\bar{X}, \mathbb{Q}_{\ell} / \mathbb{Z}_{\ell}(n-1)\right)_{G_{k}}$ is finite for $i \neq 2(n-1)$ by the Weil conjectures, which shows $c)$.

If we replace $H^{i}\left(\bar{X}, \mathbb{Q}_{\ell} / \mathbf{Z}_{\ell}(n)\right)$ by $\tilde{H}^{i}\left(\bar{X}, \mathbb{Q}_{\ell} / \mathbf{Z}_{\ell}(n)\right)$ in (32), then $\operatorname{Ker} \alpha_{1}^{2 d-i, d+1-n}$ is replaced by $\operatorname{Ker} \tilde{\alpha}_{1}^{2 d-i, d+1-n}$, since (33) induces an isomorphism

$$
\operatorname{Hom}\left(\tilde{H}^{i}\left(X, \mathbf{Q}_{\ell} / \mathbb{Z}_{\ell}(n)\right), \mathbb{Q}_{\ell} / \mathbb{Z}_{\ell}(1)\right) \cong \tilde{H}^{2 d-i}\left(\bar{X}, \mathbb{Z}_{\ell}(d+1-n)\right) .
$$

Furthermore, the last group in (32) is replaced by $\tilde{H}^{i}\left(\bar{X}, \mathbb{Q}_{\ell} / \mathbb{Z}_{\ell}(n-1)\right)_{G_{k}}$, which is zero for $i \neq 2(n-1)$, as a finite and $\ell$-divisible group. Finally, by Tate's local duality theorem [Ta1, 2.1] and (34) we get

$$
H^{2}\left(G_{\mathfrak{p}}, \tilde{H}^{i}\left(\bar{X}, \mathbf{Q}_{\ell} / \mathbb{Z}_{\ell}(n)\right)\right) \cong \tilde{H}^{i}\left(\bar{X}, \mathbf{Q}_{\ell} / \mathbb{Z}_{\ell}(n-1)\right)_{G_{\mathfrak{p}}}
$$


for all primes $\mathfrak{p}$. For $\mathfrak{p} \notin S$ this is zero for $i \neq 2(n-1)$ by the Weil conjectures and the same arguments as above (good reduction, $\mathfrak{p} \nmid \ell$ ). Thus d) follows from $b$ ).

Corollary 4. $H^{2}\left(G_{k}, \tilde{H}^{i}\left(\bar{X}, Q_{\ell} / \mathbb{Z}_{\ell}(n)\right)\right)=0$ (equivalently, $r_{i, n}^{\prime}=0$ ) for almost all $n \in \mathbb{Z}$.

Proof: This follows from Theorem $3 \mathrm{~d}$ ) and (36), since obviously $\tilde{H}^{i}\left(\bar{X}, Q_{\ell} / \mathbb{Z}_{\ell}(n-1)\right)_{G_{\mathrm{p}}} \neq 0$ only for finitely many $n$ (at most $\operatorname{dim}_{Q_{\ell}} H^{i}\left(\bar{X}, Q_{\ell}\right)$ many for fixed $\mathfrak{p}$ ).

For the Tate module $T_{\ell} A$ of an abelian variety $A$, the injectivity of the localization map $H^{1}\left(G_{k}, T_{\ell} A\right) \rightarrow \prod_{\mathfrak{p}} H^{1}\left(G_{\mathfrak{p}}, T_{\ell} A\right)$ has been shown by Wake, by a different method (see [Wak] and also [Mi2, I p. 109 ff.]). Conjecturally, $\operatorname{Ker} \alpha_{1}^{i, n}$ and $\operatorname{Ker} \alpha_{2}^{i, n}$ are finite for all $n \in \mathbb{Z}$. Namely, Grothendieck and Serre have conjectured that the action of $G_{k}$ on $H^{i}\left(\bar{X}, Q_{\ell}\right)$ is semisimple, and one has

LEMMA 10. If the action of $G_{k}$ on $H^{i}\left(\bar{X}, Q_{\ell}\right)$ is semi-simple, then the localization maps $\alpha_{1}^{i, n}$ and $\alpha_{2}^{i, n}$ have finite kernels for all $n \in \mathbb{Z}$.

Proof: We have only to consider $\alpha_{1}$, since then the case of $\alpha_{2}$ follows by the global duality theorem. Let $M=H^{i}\left(\bar{X}, \mathbb{Z}_{\ell}(n)\right)$ and

$$
M^{\delta}=\bigcup_{K / k} M^{G_{K}},
$$

where $K$ runs over all finite extensions of $k$. By the assumed semi-simplicity, the exact sequence

$$
0 \rightarrow M^{\delta} \underset{r}{\stackrel{i}{\rightleftarrows}} M \rightarrow M / M^{\delta} \rightarrow 0
$$

has a rational retraction, i.e., a map $r$ as indicated with $r \circ i=\ell^{\nu}$ for some $\nu \geq 0$, and one has $\left(M / M^{\delta}\right)^{\delta}=0$. By the existence of $r$, it suffices to show that $\alpha_{1}$ has a finite kernel for $M^{\delta}$ and for $M / M^{\delta}$ (the kernels are always finitely generated over $\mathbb{Z}_{\ell}$ ). By passing to a finite extension of $k$ and using the Hochschild-Serre spectral sequence, one easily reduces to the case where $G_{k}$ acts trivially on $M^{\delta}$. But $\alpha_{1}$ is even injective for the module $\mathbb{Z}_{\ell}$, since $G_{k}$ is generated by the $G_{\mathfrak{p}}$ for $\mathfrak{p} \in P$. On the other hand, let $\mathcal{G}=\operatorname{Im}\left(G_{k} \stackrel{\rho}{\rightarrow} \operatorname{Aut}\left(M / M^{\delta}\right)\right)$ and let $\mathfrak{g}$ be the Lie algebra of $\mathcal{G}$. Then 
$\left(\left(M / M^{\delta}\right) \otimes \mathbb{Q}_{\ell}\right)^{\mathfrak{g}}=\left(\left(M / M^{\delta}\right) \otimes \mathbb{Q}_{\ell}\right)^{\delta}$ (see [L, V 2.4.10], and this space is zero as remarked above. Since $\mathfrak{g}$ is semi-simple by assumption this implies $H^{r}\left(\mathfrak{g},\left(M / M^{\delta}\right) \otimes Q_{\ell}\right)=0$ for all $r \geq 0$, see [ChE, Theorem 24.1], and we may proceed as in the proof of Theorem 3 a).

\section{The local case.}

We now investigate the local groups $H^{2}\left(G_{\mathfrak{p}}, \tilde{H}^{i}\left(\bar{X}, \mathbb{Q}_{\ell} / \mathbb{Z}_{\ell}(n)\right)\right)$.

LEMma 11. a) Let $m=i+1-n$; then

$$
\begin{aligned}
& \operatorname{dim} H^{2}\left(G_{\mathfrak{p}}, H^{i}\left(\bar{X}, \mathbb{Q}_{\ell} / \mathbb{Z}_{\ell}(n)\right)\right)=\operatorname{dim}_{Q_{\ell}} H^{2}\left(G_{\mathfrak{p}}, H^{i}\left(\bar{X}, \mathbf{Q}_{\ell}(n)\right)\right) \\
& =\operatorname{dim}_{Q_{\ell}} H^{i}\left(\bar{X}, \mathbb{Q}_{\ell}(n-1)\right)_{G_{\mathfrak{p}}}=\operatorname{dim}_{Q_{\ell}} H^{i}\left(\bar{X}, \mathbf{Q}_{\ell}(m)\right)^{G_{\mathfrak{p}}} .
\end{aligned}
$$

b) If $\mathfrak{p} \nmid \ell$, then $\operatorname{dim}_{\mathbb{Q}_{\ell}} H^{i}\left(\bar{X}, \mathbb{Q}_{\ell}(m)\right)^{G_{\mathfrak{p}}} \leq-\operatorname{ord}_{s=m} L_{\mathfrak{p}}\left(H^{i}\left(\bar{X}, \mathbf{Q}_{\ell}\right), s\right)$, with equality if $\operatorname{Fr}_{\mathfrak{p}}$ acts semi-simply on $H^{i}\left(\bar{X}, Q_{\ell}\right)^{I_{p}}$.

Proof: a) The group $G_{\mathfrak{p}}$ satisfies the property $\left(F_{\ell}\right)$ and hence all conclusions of Remark 1. This shows the first equality. The second one follows from (36), and the third one from the isomorphisms

$$
H^{i}\left(\bar{X}, Q_{\ell}(n-1)\right)^{\vee} \cong H^{2 d-i}\left(\bar{X}, Q_{\ell}(d+1-n)\right) \cong H^{i}\left(\bar{X}, Q_{\ell}(i+1-n)\right)
$$

obtained from Poincaré duality and hard Lefschetz.

b) follows from the relation

$$
\begin{aligned}
& -\underset{s=m}{\operatorname{ord}} L\left(H^{i}\left(\bar{X}, Q_{\ell}\right), s\right)=\underset{s=m}{\operatorname{ord}} \operatorname{det}\left(1-\operatorname{Fr}_{\mathfrak{p}}(N \mathfrak{p})^{-s} \mid H^{i}\left(\bar{X}, Q_{\ell}\right)^{I_{\mathfrak{p}}}\right) \\
& =\underset{s=0}{\operatorname{ord}} \operatorname{det}\left(1-\operatorname{Fr}_{\mathfrak{p}}(N \mathfrak{p})^{-s} \mid H^{i}\left(\bar{X}, Q_{\ell}\right)^{I_{\mathfrak{p}}}(m)\right)
\end{aligned}
$$

(note that $\operatorname{Fr}_{\mathfrak{p}}$ acts on $\mathbb{Q}_{\ell}(m)$ via multiplication by $(N \mathfrak{p})^{-m}$ ). Hence this number is the multiplicity of the eigenvalue 1 for $\operatorname{Fr}_{\mathfrak{p}}$ on $H^{i}\left(\bar{X}, Q_{\ell}(m)\right)^{I_{p}}$, while $H^{i}\left(\bar{X}, \mathbb{Q}_{\ell}(m)\right)^{G_{\mathrm{p}}}=\left(H^{i}\left(\bar{X}, \mathbb{Q}_{\ell}(m)\right)^{I_{\mathfrak{p}}}\right)^{\left\langle\mathrm{Fr}_{\mathrm{p}}\right\rangle}$ is the space of eigenvectors for this eigenvalue.

By Lemma 5 and Theorem 3, or directly by the exact sequence of Tate's duality theorem (compare (32))

$$
\begin{aligned}
H^{2}\left(G_{S}, H^{i}\left(\bar{X}, \mathbb{Q}_{\ell} / \mathbb{Z}_{\ell}(n)\right)\right) \rightarrow \bigoplus_{\mathfrak{p} \in S} H^{2}\left(G_{\mathfrak{p}}, H^{i}\left(\bar{X}, \mathbb{Q}_{\ell} / \mathbb{Z}_{\ell}(n)\right)\right) & \\
& \rightarrow H^{i}\left(X, \mathbb{Q}_{\ell} / \mathbb{Z}_{\ell}(n-1)\right)_{G_{S}} \rightarrow 0
\end{aligned}
$$

and the known case of $H^{2}\left(G_{\mathfrak{p}}, H^{i}\left(\bar{X}, \mathbb{Q}_{\ell} / \mathbb{Z}_{\ell}(n)\right)\right)$ for $\mathfrak{p} \notin S$ we see that Conjecture 1 implies the local 
Conjecture 3. $H^{2}\left(G_{\mathfrak{p}}, H^{k}\left(\bar{X}, \mathbb{Q}_{\ell}(n)\right)\right)=0$ if

a) $i+1<n$, or

b) $i+1>2 n$.

(Equivalent formulation by Lemma 11: $H^{i}\left(\bar{X}, Q_{\ell}(m)\right)^{G_{p}} \neq 0$ at most for $0 \leq m \leq \frac{i+1}{2}$.)

The investigation of this divides into two cases.

Case $\mathfrak{p} \nmid \ell$ : The case of good reduction has been treated above. Similarly, one has

LEMMA 12. If $X$ has potentially good reduction at $\mathfrak{p}$, then $H^{2}\left(G_{\mathfrak{p}}, \tilde{H}^{i}(\bar{X}\right.$, $\left.\left.Q_{\ell} / \mathbf{Z}_{\ell}(n)\right)\right)=0$ for $i \neq 2(n-1)$.

Proof: Since $c d_{\ell}\left(G_{\mathfrak{p}}\right) \leq 2$, the corestriction is surjective on $H^{2}$, so we may pass to some finite extension, where $X$ has good reduction and where the result follows from the Weil conjecture.

In general, we may pass to some open subgroup $G_{\mathfrak{p}}^{\prime}$ of $G_{\mathfrak{p}}$ such that the action of its ramification group on $V=H^{i}\left(\bar{X}, Q_{\ell}\right)$ becomes trivial and the action of its inertia group $I_{\mathfrak{p}}^{\prime}$ becomes unipotent (see [SGA 7 I]). Let $M$. be the associated monodromy filtration, cf. [D4, 1.6.1]. This is a finite, ascending, $G_{\mathfrak{p}}$-equivariant filtration on $V$ such that $I_{\mathfrak{p}}^{\prime}$ acts trivially on $\mathrm{Gr}_{r}^{M} V=M_{r} V / M_{r-1} V$ for all $r \in \mathbb{Z}$. There is the well-known

Monodromy Conjecture (see [D1] and [RZ, Introduction]). One has

$$
0=M_{-i-1} V \subseteq M_{-i} V \subseteq \cdots \subseteq M_{i-1} V \subseteq M_{i} V=V,
$$

and $\mathrm{Gr}_{r}^{M} V$ is pure of weight $i+r$ (with respect to the action of the Frobenius in $G_{\mathfrak{p}}^{\prime} / I_{\mathfrak{p}}^{\prime}$; this does not depend on the choice of $G_{\mathfrak{p}}^{\prime}$ and can be reformulated purely in terms of $G_{\mathfrak{p}}$, cf. $\left.[\mathrm{D} 4,1.7]\right)$.

The function field analogue was proved by Deligne [D4, 1.8.4]. For number fields the conjecture is known for abelian varieties; for further results and discussions see [RZ].

By definition of $M_{\bullet}$ one has

$$
V^{I_{\mathrm{p}}} \subseteq M_{0} V
$$

hence the Monodromy Conjecture would imply that this $G_{\mathfrak{p}} / I_{\mathfrak{p}}$-representation is mixed with weights between 0 and $i$. In particular, this would imply the following, well-known conjecture (cf. [D3, p. 319]): 
Conjecture (on the bad factors of the $L$-function). $L_{\mathfrak{p}}\left(H^{i}\left(\bar{X}, \mathbb{Q}_{\ell}\right), s\right)$ has poles at most for $0 \leq \operatorname{Re}(s) \leq \frac{i}{2}$.

This conjecture has been proved in many cases; by Lemma $11 \mathrm{~b}$ ) it implies Conjecture 3 for $\mathfrak{p} \nmid \ell$ (in fact, we only need the case $s=m \in \mathbb{Z}$ ).

Case $\mathfrak{p} \mid \ell=p$ : Let $\mathbb{C}_{p}$ be the completion of the algebraic closure of $Q_{p}$. Faltings [Fal] has proved the Hodge-Tate decomposition

$$
H^{i}\left(\bar{X}, \mathbb{Q}_{p}\right) \underset{\mathbb{Q}_{p}}{\bigotimes} \mathbb{C}_{p} \cong \underset{r+t=i}{\bigoplus} H^{t}\left(X, \Omega_{X}^{r}\right) \underset{k}{\bigotimes} \mathbb{C}_{p}(-r)
$$

depending on an embedding $\bar{k} \hookrightarrow \mathbb{C}_{p}$ inducing $\mathfrak{p}$. This is an isomorphism of topological $G_{\mathfrak{p}}$-modules, and Tate has proved

$$
\mathbb{C}_{p}(\nu)^{G_{\mathfrak{p}}}= \begin{cases}k_{\mathfrak{p}}(\text { completion of } k \text { at } \mathfrak{p}), & \nu=0 \\ 0, & \nu \neq 0\end{cases}
$$

see [Ta2, Theorem 2].

Corollary 5 (compare [Sou4, Theorem 2 iii]). For $\mathfrak{p} \mid p$ one has

$$
\left(\begin{array}{ll}
H^{i}\left(\bar{X}, \mathbb{Q}_{p}(m)\right) \underset{\mathbb{Q}_{p}}{\bigotimes} \mathbb{C}_{p}
\end{array}\right)^{G_{\mathrm{p}}}=0 \text { for } \quad \begin{aligned}
& \text { a) } m<0 \quad(\Longleftrightarrow i+1<n), \text { or } \\
& \left.b^{\prime}\right) m>i \quad(\Longleftrightarrow n<1) .
\end{aligned}
$$

In particular, the same vanishing holds for $H^{i}\left(\bar{X}, \mathbb{Q}_{p}(m)\right)^{G_{p}} \subseteq\left(H^{i}(\bar{X}\right.$, $\left.\left.\mathbb{Q}_{p}(m)\right) \otimes \mathbb{C}_{p}\right)^{G_{\mathfrak{p}}}$, and, by Lemma $11, H^{2}\left(G_{\mathfrak{p}}, \tilde{H}^{i}\left(\bar{X}, \mathbb{Q}_{\ell} / \mathbb{Z}_{\ell}(n)\right)\right)$ vanishes for the indicated values of $n=i+1-m$.

To obtain a better bound than $b^{\prime}$ ), we consider the finer, crystalline theory. First assume that $X$ has good reduction at $\mathfrak{p}$. Let $\mathcal{X}(\mathfrak{p})$ be a smooth and proper model of $X$ over $\mathcal{O}_{\mathfrak{p}}$, the ring of integers in $k_{\mathfrak{p}}$, and let $\mathcal{X}_{\mathfrak{p}}=\mathcal{X}(\mathfrak{p}) \times_{\mathcal{O}_{p}} \kappa(\mathfrak{p})$ be the special fiber, where $\kappa(\mathfrak{p})$ is the residue field of $\mathcal{O}_{\mathfrak{p}}$. Let $k_{\mathfrak{p}}^{0}$ be the maximal unramified extension of $Q_{p}$ in $k_{\mathfrak{p}}$ and let $\mathcal{O}_{\mathfrak{p}}^{0}$ be its ring of integers (note that $\mathcal{O}_{\mathfrak{p}}^{0}=W(\kappa(\mathfrak{p})$ ) is the ring of Witt vectors for $\kappa(\mathfrak{p}))$. Then one has the

Crystalline Conjecture (Fontaine [Fo2, A.11]). There are canonical $G_{p}$-isomorphisms

$$
H^{i}\left(\bar{X}, \mathbb{Q}_{p}(m)\right) \cong\left\{v \in H_{\text {cris }}^{i}\left(\mathcal{X}_{\mathfrak{p}} / \mathcal{O}_{\mathfrak{p}}^{0}\right) \bigotimes_{\mathcal{O}_{\mathfrak{p}}^{0}} B_{\text {cris }} \mid \phi v=p^{m} v\right\} \cap F^{m}
$$


for all $i \geq 0$ and $m \in \mathbb{Z}$.

Here $H_{\text {cris }}^{i}$ denotes the crystalline cohomology, and $B_{\text {cris }}=B_{\text {cris }}\left(k_{\mathfrak{p}}\right)$ is a certain ring containing $k_{\mathfrak{p}}^{0}$ equipped with three compatible structures:

- an action of $G_{p}$

- a decreasing filtration $F^{\bullet}$ on $B_{\text {cris }} \otimes_{k_{\mathrm{p}}^{0}} k_{\mathfrak{p}}$

- a Frobenius endomorphism $\phi$ inducing the absolute Frobenius $\sigma$ on $k_{\mathrm{p}}^{0}$.

The Frobenius $\phi$ on $H_{\text {cris }}^{i} \otimes B_{\text {cris }}$ is the tensor product of $\phi$ on $B_{\text {cris }}$ and the crystalline Frobenius $\phi$ on $H_{\text {cris }}^{i}$; this makes sense, since both are $\sigma$ linear. The filtration $F^{\bullet}$ in the Crystalline Conjecture is naturally defined on $\left(H_{\text {cris }}^{i} \otimes B_{\text {cris }}\right) \otimes_{k_{\mathrm{p}}^{0}} k_{\mathfrak{p}} \cong\left(H_{\text {cris }}^{i} \otimes_{\mathcal{O}_{\mathrm{p}}^{\circ}} k_{\mathfrak{p}}\right) \otimes_{k_{\mathrm{p}}}\left(B_{\text {cris }} \otimes_{k_{\mathrm{p}}^{0}} k_{\mathfrak{p}}\right)$, as the tensor product of the filtration $F^{\bullet}$ on $B_{\text {cris }} \otimes_{k_{p}^{0}} k_{p}$ and the Hodge filtration, via the canonical isomorphism with the de Rham cohomology

$$
H_{\text {cris }}^{i}\left(\mathcal{X}_{\mathfrak{p}} / \mathcal{O}_{\mathfrak{p}}^{0}\right) \underset{\mathcal{O}_{\mathfrak{p}}^{0}}{\bigotimes} k_{\mathfrak{p}} \cong H_{D R}^{i}\left(X \times_{k} k_{\mathfrak{p}} / k_{\mathfrak{p}}\right)
$$

see $[\mathrm{BO}]$.

Let $\phi_{\mathfrak{p}}=\phi^{\left[\kappa(\mathfrak{p}): \mathbb{F}_{\mathfrak{p}}\right]}$ be the Frobenius with respect to $\kappa(\mathfrak{p})$. Then $\phi_{\mathfrak{p}}$ acts $k_{\mathfrak{p}}^{0}$-linearly on $H_{\mathrm{cris}}^{i}\left(\mathcal{X}_{\mathfrak{p}} / \mathcal{O}_{\mathfrak{p}}^{0}\right) \otimes_{\mathcal{O}_{\mathfrak{p}}^{0}} k_{\mathfrak{p}}^{0}$, and it is known that the eigenvalues of $\phi_{\mathfrak{p}}$ on this space are the same as the eigenvalues of the (geometric) Frobenius $\operatorname{Fr}_{\mathfrak{p}}$ on $H_{\text {ét }}^{i}\left(\overline{\mathcal{X}}_{\mathfrak{p}}, Q_{\ell}\right)$ for $\ell \neq p$, where $\overline{\mathcal{X}}_{\mathfrak{p}}=\mathcal{X}_{\mathfrak{p}} \times_{\kappa(\mathfrak{p})} \overline{\kappa(\mathfrak{p})}$ for an algebraic closure $\overline{\kappa(\mathfrak{p})}$ of $\kappa(\mathfrak{p})$, see [KM]. On the other hand, one has

$$
\left(F^{\nu} B_{\text {cris }}\right)^{G_{\mathfrak{p}}}= \begin{cases}k_{\mathfrak{p}}^{0}, & \nu \leq 0 \\ 0, & \nu>0 .\end{cases}
$$

In particular, the Crystalline Conjecture would imply

$$
H^{i}\left(\bar{X}, \mathbb{Q}_{p}(m)\right)^{G_{\mathfrak{p}}} \subseteq\left\{v \in H_{\mathrm{cris}}^{i}\left(\mathcal{X}_{\mathfrak{p}} / \mathcal{O}_{\mathfrak{p}}^{0}\right) \otimes k_{\mathfrak{p}}^{0} \mid \phi_{\mathfrak{p}} v=(N \mathfrak{p})^{m} v\right\}
$$

Combining this with the (proved!) Weil conjectures, we would obtain

$$
H^{i}\left(\ddot{X}, Q_{\mathfrak{p}}(m)\right)^{G_{p}}=0 \text { for } i \neq 2 m
$$

as in the $\ell$-adic case of good reduction.

If $k_{\mathfrak{p}}=k_{\mathfrak{p}}^{0}$, the Crystalline Conjecture has been proved by Fontaine and Messing (for $p>\operatorname{dim} X$, see $[\mathrm{FM}]$ ) and Faltings (in general, [Fa2]). Hence we have 
Corollary 6. If $\mathfrak{p}$ is unramified in $k / \mathrm{Q}$ and $X$ has good reduction at $\mathfrak{p} \mid p$, then $H^{i}\left(\bar{X}, Q_{p}(m)\right)^{G_{\mathrm{p}}}=0$ for $i \neq 2 m$.

For arbitrary $X$ there does not yet exist a "crystalline conjecture". I think that some analogue of the Monodromy Conjecture should hold. There is an obvious guess: that there is an $G_{\mathfrak{p}}$-equivariant filtration

$$
0=M_{-i-1} V_{p} \subseteq M_{-i} V_{p} \subseteq \cdots \subseteq M_{i-1} V_{p} \subseteq M_{i} V_{p}=V_{p} \text { of } V_{p}=H^{i}\left(\bar{X}, \mathbb{Q}_{p}\right)
$$

such that $\mathrm{Gr}_{r}^{M} V_{p}$ is potentially crystalline [Fo2, 5.6], and over a finite extension of $k_{\mathfrak{p}}$ the polynomial of the "crystalline" Frobenius on the associated filtered module [Fo2, 5.1] is the same as the polynomial of the Frobenius on $\operatorname{Gr}_{r}^{M} V_{\ell}$ for the corresponding $\ell$-adic representations $V_{\ell}=H^{i}\left(\bar{X}, Q_{\ell}\right)$ for $\ell \neq p$. However, this guess is false, as can be seen in the following counterexample, due to Mazur, Tate and Teitelbaum.

Let $\omega_{4}(z)=(\eta(z) \eta(5 z))^{4}=q-4 q^{2}+2 q^{3}-5 q^{5}-\ldots, q=e^{2 \pi i z}$, where $\eta(z)$ is the Dedekind $\eta$-function. This is a new form of weight 4 of "Haupttyp" on $\Gamma_{0}(5)$ and the associated compatible system $V=\left\{V_{\ell}\right\}$ of two-dimensional $\ell$-adic representations of $G_{Q}$ [D2] has the following properties [MTT, I $\S 12$, II §15]:

i) the image of $G_{5}$ in $G l\left(V_{5}\right) \cong G l_{2}\left(Q_{5}\right)$ is open,

ii) for $\ell \neq 5, V_{\ell}^{I_{5}}$ is one-dimensional, and the Frobenius $\mathrm{Fr}_{5}$ acts on it by multiplication by -5 .

For ii) we have combined the facts that $a_{5}=-5$ in the $q$-series $\omega_{4}=$ $\sum_{n \geq 1} a_{n} q^{n}$ and that the $L$-function of $V$ equals the $L$-function of $\omega_{4}$ at all places (see [C]; indeed, the assertion needed for ii) was already proved by Langlands in his Antwerp article and, independently, by Deligne in his letter to Piatetski-Shapiro).

Consequently, $V_{\ell}$ has a non-trivial monodromy filtration for the decomposition group $G_{5}$ for all $\ell \neq 5$, while $V_{5}$ has no non-trivial $G_{5}$-filtration at all; the same holds over any finite extension of $Q$. (The $\ell$-adic Monodromy Conjecture is true here, by the way; since $\operatorname{det} V_{\ell} \cong Q_{\ell}(-3), \operatorname{Fr}_{5}$ must act by multiplication with -25 on the one-dimensional space $V_{\ell_{I_{5}}}$ for $\ell \neq 5$.) To see the connection with the subject of this paper, note that $\left\{V_{\ell}\right\}$ is a direct factor of $\left\{H^{3}\left(\bar{X}, Q_{\ell}\right)\right\}$ for a certain three-dimensional, smooth projective variety $X$ over $Q$ [D2].

This example is useful for further observations. One can also show:

iii) $V$ is unramified outside 5 (i.e., each $V_{\ell}$ is unramified outside 5 and $\ell$; this follows from the construction in [D2]), 
iv) the image of $G_{\mathbb{Q}\left(\mu_{5}\right)}$ in $G l\left(V_{5}\right)$ is a pro-5-group [Ma2].

We deduce from this:

Lemma 13. Let $k$ be a finite extension of $Q$, and let $\Lambda_{\ell} \subseteq V_{\ell}$, for each $\ell$, be a $G_{k}$-invariant lattice (i.e., $\Lambda_{\ell} \cong \mathbb{Z}_{\ell}^{2}$ as a $\mathbb{Z}_{\ell}$-module, with $\Lambda_{\ell} \otimes_{\mathbb{Z}_{\ell}} \mathbb{Q}_{\ell}=V_{\ell}$ ).

a) $V_{\ell}(\nu)^{G_{\mathfrak{p}}}=\mathbb{Q}_{\ell}$, if $\nu=1, \ell \neq 5, \mathfrak{p} \mid 5$, and the degree $f(\mathfrak{p} \mid$ $5)$ of $\mathfrak{p}$ over 5 is even; $V_{\ell}(\nu)^{G_{\mathfrak{p}}}=0$, otherwise. In particular, $H^{2}\left(G_{k}, V_{\ell} / \Lambda_{\ell}(\nu)\right)=0$ for $\nu \neq 3$, and $H^{2}\left(G_{k}, V_{5} / \Lambda_{5}(3)\right)=0$.

b) If $Q\left(\mu_{3}\right) \subseteq k$, then $\operatorname{dim} H^{2}\left(G_{k}, V_{\ell} / \Lambda_{\ell}(3)\right)>0$ for $\ell \neq 5$.

c) Let $k=\mathbb{Q}\left(\mu_{3}\right)$. If $S \supseteq S_{5}$, then $H^{2}\left(G_{S}, V_{5}(\nu)\right)=0$ for all $\nu \in \mathbb{Z}$. If $\ell \neq 5$ and $S \supseteq S_{5} \cup S_{\ell}$, then $\operatorname{dim}_{Q_{\ell}} H^{2}\left(G_{S}, V_{\ell}(3)\right) \geq 1$. In particular, $\operatorname{dim}_{Q_{5}} H^{1}\left(G_{k}, V_{5}(3)\right)=2$, but $\operatorname{dim}_{Q_{\ell}} H^{1}\left(G_{k}, V_{\ell}(3)\right)>2$ for $\ell \neq 5$.

Proof: a) The case $\mathfrak{p} \nmid 5, \mathfrak{p} \nmid \ell$ is clear by good reduction and the Weil conjectures. For $\mathfrak{p} \nmid 5$ and $\mathfrak{p} \mid \ell$ the $G_{\mathfrak{p}}$-representation $V_{\ell}$ is crystalline, since this is the case for $k=Q$ by Fontaine-Messing-Faltings, and the associated admissible filtered module is the base extension of that for $k=\mathbb{Q}$ (cf. [Fo1, 7.3.2]). Hence we may argue using the Weil conjectures as in Corollary 6. For $\mathfrak{p} \mid 5$ we have $V_{5}(\nu)^{G_{\mathfrak{p}}}=0$ for all $\nu \in \mathbb{Z}$, since the image of $G_{\mathfrak{p}}$ in $\operatorname{Aut}\left(\Lambda_{5}\right) \cong G l_{2}\left(\mathbb{Z}_{5}\right)$ is open by i). For $\mathfrak{p} \mid 5 \neq \ell$ we have $V_{\ell}(\nu)^{G_{\mathfrak{p}}}=\left\{v \in V_{\ell}^{I_{\mathfrak{p}}} \mid \operatorname{Fr}_{\mathfrak{p}} v=(N \mathfrak{p})^{\nu} \cdot v\right\}$; by ii) this is non-zero if and only if $\nu=1$ and $\operatorname{Fr}_{\mathfrak{p}}$ is an even power of $\mathrm{Fr}_{5}$. The other assertions of a) now follow from the analogues of Lemma 11 a) and Theorem $3 \mathrm{~d}$ ) for $V_{\ell}$; recall that $V_{\ell}$ is a direct factor of $H^{3}\left(\bar{X}, Q_{\ell}\right)$, moreover, (37) can be replaced by the isomorphism $V_{\ell}^{\vee} \cong V_{\ell}(3)$ (note that $\left(R^{1} f_{*} Q_{\ell}\right)^{\vee} \cong R^{1} f_{*} Q_{\ell}(1)$ in $[\mathbf{D} 4$, 3.7] by Poincaré duality).

b) follows similarly from the local results in a), since $f(\mathfrak{p} \mid 5)=2$ for the prime $\mathfrak{p} \mid 5$ in $\mathbb{Q}\left(\mu_{3}\right)$.

For the first assertion of c) note that the action of $G_{S_{5}}\left(Q\left(\mu_{15}\right)\right)$ factors through its maximal pro-5-quotient $\tilde{G}$ by iii), and that this quotient is pro5 -free by [ $\mathrm{Br}, 3.3]$, since there is only one prime above 5 in $\mathrm{Q}\left(\mu_{15}\right)$ and the class number of $\mathbb{Q}\left(\mu_{15}\right)$ is 1 [Wa1, Tables]. We obtain the vanishing of $H^{2}\left(G_{S_{5}}\left(Q\left(\mu_{15}\right)\right), V_{5} / \Lambda_{5}(\nu)\right)$, since the inflation

$$
H^{2}\left(\tilde{G}, V_{5}, \Lambda_{5}(\nu)\right) \underset{\sim}{\stackrel{\inf }{\longrightarrow}} H^{2}\left(G_{S_{5}}\left(Q\left(\mu_{15}\right)\right), V_{5} / \Lambda_{5}(\nu)\right)
$$

is an isomorphism (cf. [Neu, Cor. 1]). By the surjectivity of the corestriction on $H^{2}$, valid since $c d_{5}\left(G_{S_{5}}\left(Q\left(\mu_{3}\right)\right) \leq 2\right.$, we get the same vanishing for 
$G_{S_{5}}$ over $\mathbb{Q}\left(\mu_{3}\right)$, hence for $G_{S}$ with $S \supset S_{5}$, cf. (13). The other results are clear from a), b) and the analogues for $V_{\ell}$ of Lemmas 1 to 5 ; we have $\chi\left(G_{S}, V_{\ell}\right)=-[k: \mathbb{Q}]$ by Lemma 2 , since the Hodge structure $V_{\mathbb{R}}$ belonging to $V$ in $H^{3}(X(\mathbb{C}), \mathbb{P})$ is of type $\{(0,3),(3,0)\}[D 3, \S 7]$.

From this example we learn that for a compatible system $V=\left\{V_{\ell}\right\}$ of $\ell$ adic representations the dimension of its Galois cohomology can get smaller for the primes $\ell$ where $V$ has bad reduction. In particular, the numbers $d_{i, n}, r_{i, n}$ and $r_{i, n}^{\prime}$ in $\S 3$ may depend on $\ell$. I would expect that they are the same for all $\ell$ where $X$ has good reduction. For $r_{i, n}^{\prime}$ this would follow from the Monodromy Conjecture and the Crystalline Conjecture, via Theorem 3. For $r_{i, n}$ (and hence $d_{i, n}$ ), compare $\S 6$.

I think that there should exist a $p$-adic analogue of the monodromy filtration on the "crystalline side", in the following sense. Consider the following category $M F_{k_{\mathfrak{p}}}^{f, N}$ : objects are weakly admissible filtered modules $D$ over $k_{\mathfrak{p}}$ (see $\left[\right.$ Fo2, 5.1]) together with a nilpotent homomorphism of $k_{\mathfrak{p}}^{0}$-modules $N: D \rightarrow D$ satisfying $\phi^{-1} N \phi=p N$ (no compatibility with the filtration $\left.F^{\bullet}\right) .^{1}$ This implies that $\phi$ respects the monodromy filtration $M_{\text {• associ- }}$ ated to $N$. Morphisms between these objects are morphisms of filtered modules which respect $N$. There should exist a category $\operatorname{Rep}_{\text {cris,N }}\left(k_{\mathfrak{p}}\right)$ of $Q_{p}$-representations of $G_{\mathfrak{p}}=\operatorname{Gal}\left(\bar{k}_{\mathfrak{p}} / k_{\mathfrak{p}}\right)$, containing the crystalline ones, and an equivalence of tensor categories between $M F_{k_{\mathrm{p}}}^{f, N}$ and $\operatorname{Rep}_{\mathrm{cris,N}}\left(k_{\mathrm{p}}\right)$. Under this equivalence of categories, the crystalline representations should correspond to those objects in $M F_{k_{\mathrm{p}}}^{f, N}$ for which $N=0$ (note that this subcategory can be identified with $M F_{k_{p}}^{f}$, the category of weakly admissible filtered modules over $\left.k_{\mathfrak{p}}\right)$. $H^{i}\left(\bar{X}, Q_{p}\right)$ should be in $\operatorname{Rep}_{c r i s, N}\left(k_{p}\right)$ potentially, i.e., over a finite extension of $k_{\mathfrak{p}}$. If $H^{i}\left(\bar{X}, Q_{p}\right)$ is in $\operatorname{Rep}_{\text {cris,N }}\left(k_{\mathfrak{p}}\right)$, and $D$ is the associated object in $M F_{k_{p}}^{f, N}$, the characteristic polynomial of $\phi_{\mathfrak{p}}$ on $\mathrm{Gr}_{r}^{M} D$ should be the same as the characteristic polynomial of $\operatorname{Fr}_{\mathfrak{p}}$ on $\mathrm{Gr}_{r}^{M} V_{\ell}$ for $V_{\ell}=H^{i}\left(\bar{X}, Q_{\ell}\right), \ell \neq p$.

\footnotetext{
${ }^{1}$ Added in proof: In a letter to the author (November 1987), J.-M. Fontaine observed a wrong normalization of $\phi$ in the first version of this paper and pointed out that the modules in $M F_{k_{\mathfrak{p}}}^{f, N}$ should just be weakly $N$-admissible: in the notation of [Fo1] this means postulating $t_{H}\left(D^{\prime}\right) \leq t_{N}\left(D^{\prime}\right)$ only for submodules $D^{\prime} \subseteq D$ respected by $N$. By developing a formalism quite similar to the one for his Crystalline Conjecture, Fontaine then gives a definition for $\operatorname{Rep}_{\mathrm{cris,N}}\left(k_{\mathfrak{p}}\right)$ and the functor to $M F_{k_{\mathrm{p}}}^{f, N}$. This leads to a precise formulation of the $p$-adic Monodromy Conjecture, and Fontaine proves it for abelian varieties.
} 
This would imply

$$
\begin{aligned}
& H^{i}\left(\bar{X}, \mathbb{Q}_{p}(m)\right)^{G_{\mathfrak{p}}}=\operatorname{Hom}_{G_{\mathrm{p}}}\left(\mathbb{Q}_{p}, H^{i}\left(\bar{X}, \mathbb{Q}_{p}(m)\right)\right) \\
& \quad \cong \operatorname{Hom}_{M F_{k_{\mathrm{p}}}^{j, N}}\left(k_{\mathfrak{p}}^{0}, D(m)\right) \cong\left\{v \in D \mid \phi v=p^{m} v, N v=0\right\} \cap F^{m}
\end{aligned}
$$

We see that the dimension of this space can be smaller than the dimension of

$$
\left\{v \in D \mid \phi v=p^{m} v, N v=0\right\}
$$

which should equal the dimension of

$$
\begin{aligned}
\left\{v \in H^{i}\left(\bar{X}, Q_{\ell}\right) \mid \operatorname{Fr}_{\mathfrak{p}} v=(N \mathfrak{p})^{m} v, N v\right. & =0\} \\
& \cong H^{i}\left(\bar{X}, \mathbf{Q}_{\ell}(m)\right)^{G_{\mathfrak{p}}} \quad \ell \neq p
\end{aligned}
$$

This would explain the phenomena discussed in Lemma 13.

The compatibility of $N$ with $F^{\bullet}$ decides whether the mondromy filtration "lifts to characteristic zero", i.e., to $V_{p}$ (compare the relation between filtrations and lifting problems for $p$-divisible groups $[\mathbf{G r}]$ ). Consider the example of the two-dimensional $G_{Q_{5}}$-representation $V_{5}$ associated to $\omega_{4}$. For the associated object $D$ of $M F_{Q_{5}}^{f, N}$ one expects a filtration

$$
0=M_{-2} D \varsubsetneqq M_{-1} D=\operatorname{Im} N=\operatorname{Ker} N=M_{0} D \varsubsetneqq M_{1} D=D
$$

such that $\phi$ acts via -5 on $\operatorname{Gr}_{-1}^{M} D$ and via -25 on $\operatorname{Gr}_{1}^{M} D$. If $N$ is compatible with $F^{\bullet}$, then $\operatorname{Ker} N$ and Coker $N$ are weakly admissible; this implies $D=$ $F^{0}=F^{1} \supsetneqq F^{2} \supsetneqq F^{3}=0$. The associated 5 -adic representation would be a non-trivial extension of $Q_{5}(-2)(\chi)$ by $Q_{5}(-1)(\chi)$, where $(\chi)$ denotes the twist by the character belonging to the unramified extension of degree 2 of $Q_{5}$. However, the Hodge filtration on $D$ should satisfy

$$
D=F_{0} \supsetneqq F^{1}=F^{2}=F^{3} \supsetneqq F^{4}=0
$$

(recall that $V_{\mathbf{R}}$ is of type $\left.\{(0,3),(3,0)\}\right)$. Then Newton polygon and Hodge polygon (see [Fo1, 4.3]) look like: 


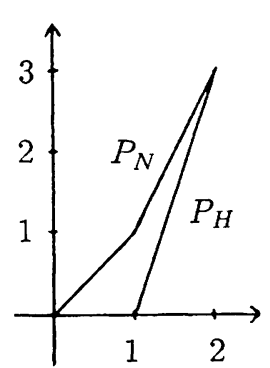

It follows from $[$ Fo1, 4.3.3] that $D$ is weakly admissible if and only if $\phi$ does not respect $F^{1} D$. It is then also admissible, since the length is $4<p=5$ [FL, Theorem 8.4]. Moreover, we see from (44) that there are no proper weakly admissible submodules, therefore the associated crystalline $G_{Q_{5}}$-representation $\tilde{V}_{5}$ is irreducible as $V_{5}$ is. Nevertheless, $\tilde{V}_{5}$ should not be isomorphic to $V_{5}$, since it corresponds to the module $D$ with $N=0 .^{2}$

REMARK 5: The discussion above suggests that one should - for all $\mathfrak{p}$ and $\ell$ - also expect $H^{2}\left(G_{\mathfrak{p}}, H^{i}\left(\bar{X}, Q_{\ell}(n)\right)\right)=0$ for $i+1=2 n$ (that is, $H^{i}\left(\bar{X}, Q_{\ell}(m)\right)^{G_{p}}=0$ for $\left.m=\frac{i+1}{2}\right)$, thus sharpening Conjecture 3. Also, everything could be formulated for varieties over $k_{\mathfrak{p}}$, not mentioning a global field, and the conjectures should still be true.

6. The case $n \leq i+1 \leq 2 n$.

Let $V$ be an $\ell$-adic representation of $G_{S}$, then the kernels of the localization maps

$$
\begin{gathered}
\alpha_{1, S}(V): H^{1}\left(G_{S}, V\right) \quad \longrightarrow \bigoplus_{\mathfrak{p} \in S} H^{1}\left(G_{\mathfrak{p}}, V\right) \\
\alpha_{2, S}\left(V^{\vee}(1)\right): H^{2}\left(G_{S}, V^{\vee}(1)\right) \longrightarrow \bigoplus_{\mathfrak{p} \in S} H^{2}\left(G_{\mathfrak{p}}, V^{\vee}(1)\right)
\end{gathered}
$$

are $Q_{\ell}$-dual tc each other by Tate's duality theorem (here $V^{\vee}$ is the $\mathbb{Q}_{\ell}$-dual of $V)$.

Question 2 Let $V$ be pure of weight $w$. Is $\alpha_{1, S}(V)$ injective for $w \neq-1$ (or, equivalently, is $\alpha_{2, S}(V)$ injective for $w \neq-1$ )?

REMARK 6: Both $G_{S}$ and the $G_{\mathfrak{p}}$ satisfy the condition $\left(F_{\ell}\right)$ of Remark 1.

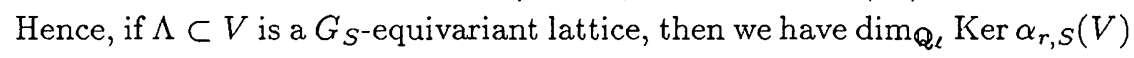

${ }^{2}$ Added in proof: In view of the previous footnote, it is possible that $V_{5}$ corresponds to the module $D=Q_{5} e_{1} \oplus \mathbb{Q}_{5} e_{2}$ with $\phi e_{1}=-5 e_{1}, \phi e_{2}=-25 e_{2}, N e_{1}=0, N e_{2}=e_{1}$, $F^{0} D=D, F^{1} D=F^{3} D=\mathbb{Q}_{5} e_{2}$, and $F^{4} D=0$, which is weakly $N$-admissible but not weakly admissible. 
$=\operatorname{dim} \operatorname{Ker} \alpha_{r, S}(V / \Lambda)=\operatorname{rank}_{\mathbb{Z}_{\ell}} \operatorname{Ker} \alpha_{r, S}(\Lambda)$, so we may equivalently ask for the finiteness of the last two kernels. Also, we may replace $\Lambda$ or $V / \Lambda$ by isogenous modules. In the commutative diagram

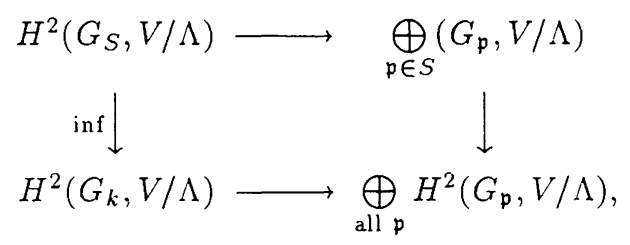

the bottom map is injective for $V$ pure of weight $w \neq-2$ by the arguments of Theorem 3. Hence for $w \neq-2$ we have $\operatorname{dim} \operatorname{Ker} \alpha_{2, S}(V / \Lambda)=$ $\operatorname{dim} \operatorname{Ker}\left(H^{2}\left(G_{S}, V / \Lambda\right) \stackrel{\text { inf }}{\rightarrow} H^{2}\left(G_{k}, V / \Lambda\right)\right)$ and may reformulate Question 2 in terms of the above inflation map. It is more complicated to describe what this implies for the $H^{2}$-inflation for $V$ or $\Lambda$.

THEOREM 4. The function field analogue of Question 2 has a positive answer for $w \neq 0,-1,-2$. More precisely, let $U$ be a smooth, affine curve over a finite field $\mathbf{F}_{q}$ and let $V$ be a $Q_{\ell}$-representation of $\pi_{1}(U, \bar{\eta})$ of weight $w$ (here $\bar{\eta}$ is a geometric point over the generic point $\eta=\operatorname{Spec} k$ of $U$ ). Let $Y$ be the smooth, projective compactification of $U$, and for each $x \in Y \backslash U$ let $G_{x} \subseteq G_{k}=\pi(\eta, \bar{\eta})$ be a decomposition group at $x$. Then the localization map

$$
\alpha_{1}: H^{1}\left(\pi_{1}(U, \bar{\eta}), V\right) \rightarrow \bigoplus_{x \in Y \backslash U} H^{1}\left(G_{x}, V\right)
$$

is injective for $w \neq-1$, except possibly when $w=0$ and $V^{G_{k}} \neq 0$, and

$$
\alpha_{2}: H^{2}\left(\pi_{1}(U, \bar{\eta}), V\right) \rightarrow \bigoplus_{x \in Y \backslash U} H^{2}\left(G_{x}, V\right)
$$

is injective for $w \neq-1$, except possibly when $w=-2$ and $V(-1)_{G_{k}} \neq 0$. If $w=-1$, then $\alpha_{1}$ and $\alpha_{2}$ are the zero maps.

Proof: Let $F$ be the smooth $\mathbb{Q}_{\ell}$-sheaf on $U$ corresponding to $V$, and let $j: U \hookrightarrow Y$ be the inclusion. Then the localization maps $\alpha_{r}$ can be identified with the maps $\alpha_{r}$ in the relative cohomology sequence

$$
\begin{aligned}
\cdots \rightarrow H^{r}\left(Y, j_{!} F\right) \stackrel{\beta_{r}}{\rightarrow} & H^{r}(U, F) \stackrel{\alpha_{r}}{\rightarrow} \\
& \bigoplus_{x \in Y^{\backslash} \backslash U} H_{x}^{r+1}\left(Y, j_{!} F\right) \rightarrow H^{r+1}\left(Y, j_{!} F\right) \rightarrow \ldots
\end{aligned}
$$


compare $[\mathrm{Mi} 2,4.13 . c]$. It remains to consider the maps

$$
\beta_{r}: H_{c}^{r}(U, F)=H^{r}\left(Y, j_{!} F\right) \rightarrow H^{r}(U, F) .
$$

Now $\beta_{r}$ factors through $H^{r}\left(Y, j_{*} F\right)$, and the Hochschild-Serre spectral sequence gives an exact sequence

$$
0 \rightarrow H^{r-1}\left(\bar{Y}, j_{*} F\right)_{\Gamma} \rightarrow H^{r}\left(Y, j_{*} F\right) \rightarrow H^{r}\left(\bar{Y}, j_{*} F\right)^{\Gamma} \rightarrow 0,
$$

$\Gamma=\operatorname{Gal}\left(\overline{\boldsymbol{F}}_{q} / \mathbf{F}_{q}\right)$ and $\bar{Y}=Y \times_{\mathbf{F}_{q}} \overline{\mathbf{F}}_{q}$. Moreover, Deligne has shown that $H^{r}\left(\bar{Y}, j_{*} F\right)$ is pure of weight $r+w$, see $[\mathbf{D} 4,3.2 .3]$. This implies the vanishing of $H^{r}\left(Y, j_{*} F\right)$ for $r+w \neq 0$ and $r-1+w \neq 0$.

For $r=1$ and $w=0$ we get the isomorphism $H^{1}\left(Y, j_{*} F\right) \cong H^{0}\left(\bar{Y}, j_{*} F\right)_{\Gamma}$; the latter group has the same dimension as

$$
H^{0}\left(\bar{Y}, j_{*} F\right)^{\Gamma}=H^{0}(U, F)=V^{G_{k}} .
$$

For $r=2$ and $w=-2$ we have $H^{2}\left(Y, j_{*} F\right) \cong H^{2}\left(\bar{Y}, j_{*} F\right)^{\Gamma} \cong$ $H^{0}\left(\bar{Y}, j_{*} F^{\vee}(1)\right)_{\Gamma}{ }^{\vee}$, see loc. cit.; this has the same dimension as

$$
H^{0}\left(\bar{Y}, j_{*} F^{\vee}(1)\right)^{\Gamma}=H^{0}\left(U, F^{\vee}(1)\right)=\operatorname{Hom}_{G_{k}}\left(V, \mathbb{Q}_{\ell}(1)\right) .
$$

Finally, for $w=-1$ and $x \in S$, we obtain

$$
H^{0}\left(G_{x}, V\right)=\left(V^{I_{x}}\right)^{\mathrm{Gal}\left(\tilde{F}_{q} / \kappa(x)\right)}=0
$$

( $I_{x} \subset G_{x}$ the inertia group), since the stalk $F_{x}=V^{I_{x}}$ has weights $\leq$ $-1[\mathrm{D} 4,1.8 .1]$. By local duality, we also have

$$
H^{2}\left(G_{k}, V\right) \cong H^{0}\left(G_{k}, V^{\vee}(1)\right)^{\vee}=0 .
$$

This implies $H^{1}\left(G_{x}, V\right)=0$, since

$$
0=\chi\left(G_{x}, N\right):=\sum_{\nu=0}^{3}(-1)^{\nu} \operatorname{dim}_{Q_{\ell}} H^{\nu}\left(G_{x}, N\right)
$$

for every $Q_{\ell}$-representation $N$ of $G_{x}$ (this follows, e.g., from [Ta1, 2.2] as in Lemma 2).

REMARK 7: If $V$ is a semi-simple $\pi_{1}(U, \bar{\eta})$-representation, we get the injectivity of $\alpha_{r}$ also for $w=0$ and $w=-2$ : If $V \cong \mathbb{Q}_{\ell}^{r} \oplus V_{1}$ with $V_{1}^{G_{k}}=0$, we 
have $\operatorname{Ker} \alpha_{1}\left(V_{1}\right)=0$ by the theorem, and $\operatorname{Ker} \alpha_{1}\left(Q_{\ell}\right)=0$, since the class group of $k$ is finite. The case of $\alpha_{2}$ follows by duality.

The weight of $H^{i}\left(\bar{X}, \mathbf{Q}_{\ell}(n)\right)$ is $i-2 n$; hence we ask for the injectivity of $\alpha_{r, S}^{i, n}:=\alpha_{r, S}\left(H^{i}\left(\bar{X}, Q_{\ell}(n)\right)\right)$ for $i \neq 2 n-1$. Conjecture 1 implies that $\alpha_{2, S}^{i, n}$ is injective for a) $i+1<n$ or b) $i+1>2 n$, hence that $\alpha_{1, S}^{i, n}$ is injective for a) $n<0$ or b) $i+1<2 n$, by using (37). Case b) would imply that we could consider the regulator maps

$$
r: K_{2 n-i-1}(X)^{(n)} \otimes \mathbb{Q}_{\ell} \rightarrow H^{1}\left(G_{S}, H^{i}\left(\bar{X}, \mathbf{Q}_{\ell}(n)\right)\right), \quad 2 n-i-1>0,
$$

locally. Conversely, a positive answer for Question 2 would imply that $r_{i, n}=r_{i, n}^{\prime}$ for $i \neq 2 n-1, i \neq 2 n-2$, hence that $r_{i, n}$ could be computed in purely local terms except for these values of $i$ and $n$. If $i=2 n-2$, then $r_{i, n}^{\prime}$ can be infinite and $r_{i, n}$ may depend on $S$, cf. Example 1 . If $i=2 n-1$, then $r_{i, n}$ is of "global nature", and $\alpha_{2}$ is expected to be zero, cf. Remark 5 and Remark 8. Similar statements hold for the numbers $d_{i, n}$, by the formula

$$
\chi_{i, n}=\operatorname{dim}_{Q_{\ell}} H^{i}\left(\bar{X}, Q_{\ell}\right)^{G_{k}}-d_{i, n}+r_{i, n},
$$

in which $H^{i}\left(\bar{X}, Q_{\ell}(n)\right)^{G_{k}}$ vanishes for $i \neq 2 n$ and is related to Tate's conjecture for $i=2 n$.

I conclude this chapter with some speculation on the regulator maps for $n \leq i+1 \leq 2 n$. I think that they are still injective, at least if one restricts to $K_{2 n-i-1}(X / \mathbb{Z})^{(n)} \otimes Q_{\ell}$, and that the image of the last group in $H^{1}\left(G_{S}, H^{i}\left(\bar{X}, Q_{\ell}(n)\right)\right)$ can be described by local conditions. (For $i+1=2 n$, the above regulator maps are defined on $K_{0}(X)_{0}^{(n)} \otimes Q_{\ell}$, where $K_{0}(X)_{0}^{(n)}=\operatorname{Ker}\left(K_{0}(X)^{(n)} \rightarrow H^{2 n}\left(\bar{X}, Q_{\ell}(n)\right)^{G_{k}}\right)$, and we consider this whole space, since $\left.K_{0}(X / \mathbb{Z})^{(n)}=K_{0}(X)^{(n)}\right)$. The nature of these local conditions is suggested by work of $\mathrm{R}$. Greenberg (on the Selmer group of a compatible system of $\ell$-adic representations), S. Bloch (on Tamagawa numbers of motives), and P. Schneider (on a $p$-adic, local version of Beilinson's conjectures, aiming at relations between $p$-adic regulators and $p$-adic $L$ functions). The subspace in question should be defined via the localization map

$$
H^{1}\left(G_{S}, H^{i}\left(\bar{X}, Q_{\ell}(n)\right)\right) \rightarrow \bigoplus_{\mathfrak{p} \in S} H^{1}\left(G_{\mathfrak{p}}, H^{i}\left(\bar{X}, Q_{\ell}(n)\right)\right)
$$


as the pre-image of a space $\oplus_{\mathfrak{p} \in S} A_{\mathfrak{p}}$, where for good reduction at $\ell$ the spaces $A_{\mathfrak{p}} \subseteq H^{1}\left(G_{\mathfrak{p}}, H^{i}\left(\bar{X}, Q_{\ell}(n)\right)\right)$ are defined as follows

$$
\begin{aligned}
& A_{\mathfrak{p}}=0 \text { for } \mathfrak{p} \nmid \ell \\
& A_{\mathfrak{p}}=H_{D R}^{i}\left(X \times k_{\mathfrak{p}} / k_{\mathfrak{p}}\right) /\left(\phi-p^{n}\right) F^{n} H_{D R}^{i}\left(X \times k_{\mathfrak{p}} / k_{\mathfrak{p}}\right) \text { for } \mathfrak{p} \mid \ell .
\end{aligned}
$$

The embedding of the last group in $H^{1}\left(G_{\mathfrak{p}},-\right)$ is obtained via the connecting morphism of the $G_{\mathfrak{p}}$-cohomology sequence associated to the exact sequence

$$
\begin{aligned}
& 0 \rightarrow H^{i}\left(\bar{X}, \mathbb{Q}_{p}(n)\right) \rightarrow F^{n}\left(H_{D R}^{i}\left(X \times k_{\mathfrak{p}} / k_{\mathfrak{p}}\right) \otimes B_{\text {cris }}\right) \\
& \stackrel{\phi-p^{n}}{\longrightarrow} H_{D R}^{i}\left(X \times k_{\mathfrak{p}} / k_{\mathfrak{p}}\right) \otimes B_{\text {cris }} \rightarrow 0
\end{aligned}
$$

derived from the Crystalline Conjecture (and proved for $k_{\mathfrak{p}}=k_{\mathfrak{p}}^{0}$ and $p>$ $\operatorname{dim} X$ by Fontaine and Messing). The idea to consider these spaces for $\mathfrak{p} \mid p$ is due to K. Kato.

\section{The case $i=1$ : abelian varieties.}

The Kummer sequences $0 \rightarrow \mu_{\ell^{r}} \rightarrow \mathbb{G}_{m} \stackrel{\ell^{r}}{\rightarrow} \mathbb{G}_{m} \rightarrow 0$ induce an isomorphism

$$
H^{1}\left(\bar{X}, \mathbb{Z}_{\ell}(1)\right) \cong T_{\ell} H^{1}\left(\bar{X}, G_{m}\right)=T_{\ell} A,
$$

where $A=\underline{\mathrm{Pic}}_{X / k}^{0}$ is the Picard variety of $X / k$. (Conversely, if we start with an abelian variety $A$, then we get such an isomorphism for $X=A^{\prime}$, the dual abelian variety). By [SGA 7 I, exp. IX, $\S 6$ f.], the local conjectures are true in this case for every prime $\mathfrak{p}$ : There is a filtration of $G_{\mathfrak{p}}$-submodules

$$
0 \subseteq T_{\ell}(A)^{\mathrm{et}} \subseteq T_{\ell}(A)^{\mathrm{ef}} \subseteq T_{\ell}(A)
$$

(et = essentially toric, ef = essentially finite), coinciding with the monodromy filtration after tensoring with $\mathbb{Q}_{\ell}$ for $\mathfrak{p} \nmid \ell$ (for the last property see [SGA 71 , exp. I, §6]), and a semi-abelian variety $A^{\text {b0 }}$ over $k_{\mathfrak{p}}$ (the connected Raynaud scheme) with the following properties: There is an exact sequence of group schemes

$$
0 \rightarrow T \rightarrow A^{\natural 0} \rightarrow B \rightarrow 0
$$


such that $T$ is a torus, $B$ is an abelian variety with potentially good reduction, and one has canonical $G_{\mathfrak{p}}$-isomorphisms

$$
\begin{aligned}
T_{\ell}(T) & \cong T_{\ell}(A)^{\mathrm{et}}, \\
T_{\ell}\left(A^{\natural 0}\right) & \cong T_{\ell}(A)^{\mathrm{el}},
\end{aligned}
$$

(all primes $\ell$ ). Moreover, there is an ismorphism

$$
T_{\ell}(A) / T_{\ell}(A)^{\mathrm{ef}} \cong \operatorname{Hom}\left(T_{\ell}\left(A^{\prime}\right)^{\mathrm{et}}, \mathbb{Z}_{\ell}(1)\right),
$$

where $A^{\prime}$ is the dual abelian variety. Hence for $\mathfrak{p} \nmid \ell, T_{\ell}(A)^{\mathrm{et}}, T_{\ell}(A)^{\mathrm{e}} /$ $T_{\ell}(A)^{\mathrm{et}} \cong T_{\ell}(B)$ and $T_{\ell}(A) / T_{\ell}(A)^{\mathrm{ef}}$ are pure of weight $-2,-1$ and 0 , respectively. For $\mathfrak{p} \mid \ell=p$, it follows from the work of Fontaine [Fo1, 3.3.5], [Fo2, 6.2] that $T_{p}(T), T_{p}(B)$ and $\operatorname{Hom}\left(T_{p}\left(T^{\prime}\right), \mathbb{Z}_{p}(1)\right)$ are potentially crystalline (here $T^{\prime}$ is the maximal subtorus of $\left(A^{\prime}\right)^{\mathrm{t0}}$ ), and that over a suitable extension of $k_{\mathfrak{p}}$ the characteristic polynomial of the crystalline Frobenius on the associated admissible filtered modules is the same as the Frobenius polynomial on the $\ell$-adic counterparts, $\ell \neq p$. By using the isogeny $T_{p}(A) / T_{p}(A)^{\mathrm{ef}} \rightarrow T_{p}(A)^{\mathrm{et}}(-1)$ coming from [SGA $7 \quad 1$, exp. IX, §9], it is even possible to define a $p$-adic monodromy operator $N=N_{p}: V_{p}(A) \rightarrow V_{p}(A)(-1)$ such that the associated monodromy filtration coincides with (48) after tensoring with $\mathbb{Q}_{p}$. Thus, in this case the "naive" form of the p-adic monodromy is present, but this shall not be needed in the following.

THEOREM 5. Let $V_{\ell}(A)=T_{\ell}(A) \otimes_{\mathbf{Z}_{\ell}} Q_{\ell}$.

a) $H^{1}\left(\bar{X}, Q_{\ell}(r+1)\right)^{G_{\mathrm{p}}} \cong V_{\ell}(A)(r)^{G_{\mathrm{p}}}=0$ for $r \neq-1$.

b) $H^{1}\left(\bar{X}, Q_{\ell}\right)^{G_{\mathfrak{p}}} \cong V_{\ell}(A)(-1)^{G_{\mathrm{p}}} \cong X_{*}(T)_{k_{\mathrm{p}}} \otimes_{\mathbb{Z}} \mathbf{Q}_{\ell}$,

where $X_{*}(T)_{k_{\mathrm{p}}}=\operatorname{Hom}_{k_{\mathrm{p}}}\left(G_{m}, T\right)$ is the group of cocharacters over $k_{\mathrm{p}}$ of $T$ (and $T$ is the torus associated to $A$ over $k_{\mathfrak{p}}$ as above). If $A$ has semi-stable reduction at $\mathfrak{p}$, then $\operatorname{rank} X_{*}(T)_{k_{\mathfrak{p}}}=\operatorname{dim} T_{s}^{0}$, where $T_{s}$ is the maximal torus of $\mathcal{A}_{s}^{0}$, the connected component of the special fibre $\mathcal{A}_{s}$ of the Neron model $\mathcal{A}$ of $A$ (over $\mathcal{O}_{k}$ or $\mathcal{O}_{p}$ ), and $T_{s}^{0} \subset T_{s}$ is the maximal split torus.

Proof: For $\mathfrak{p} \nmid \ell$ one has $T_{\ell}(A)(r)^{G_{\mathfrak{p}}} \subseteq T_{\ell}(A)(r)^{I_{\mathfrak{p}}} \subseteq T_{\ell}(A)^{\mathrm{ef}}(r)$ (coincidence of (48) with the monodromy filtration). Now $T_{\ell}(B)(r)^{G_{\mathrm{p}}}=0$ for all $r \in \mathbf{Z}$ and $T_{\ell}(T)(r)^{G_{\mathrm{p}}}=0$ for $r \neq-1$ by questions of weights, hence we get a) and

$$
\begin{aligned}
T_{\ell}(A)(-1)^{G_{\mathrm{p}}}=T_{\ell}(A)^{\mathrm{ef}}(-1)^{G_{\mathrm{p}}}=T_{\ell}(T)(-1)^{G_{\triangleright}} & \\
& =\operatorname{Hom}_{G_{\mathrm{p}}}\left(T_{\ell} G_{m}, T_{\ell} T\right)=\operatorname{Hom}_{k_{\mathrm{p}}}\left(G_{m}, T\right) \underset{\mathbb{Z}}{\mathbb{Z}_{\ell}}
\end{aligned}
$$


by the theory of tori.

For $\mathfrak{p} \mid \ell=p$, we use the Hodge-Tate decomposition

$$
H^{1}\left(\bar{X}, Q_{p}\right) \underset{\mathbb{Q}_{p}}{\otimes} \mathbb{C}_{p} \cong H^{1}\left(X, \mathcal{O}_{X}\right) \underset{k}{\otimes} \mathbb{C}_{p} \oplus H^{0}\left(X, \Omega_{X}^{1}\right) \underset{k}{\otimes} \mathbb{C}_{p}(-1)
$$

as in Corollary 5 to deduce $H^{1}\left(\bar{X}, Q_{p}\right)(r+1)^{G_{p}}=0$ for $r \neq 0,-1$. For $r=0$ we have $T_{p}(A)^{G_{\mathrm{p}}}=T_{p}\left(A\left(k_{\mathfrak{p}}\right)\right)=0$, since the $p$-torsion in $A\left(k_{\mathfrak{p}}\right)$ is finite. For $r=-1$ we first observe that $\left(T_{p}(A) / T_{p}(A)^{\mathrm{ef}}(-1)\right)^{G_{\mathrm{p}}}=0$, since $G_{\mathfrak{p}}$ acts on $T_{p}(A) / T_{p}(A)^{\text {ef }}$ via a finite quotient by (51). Furthermore, $T_{p}(B)(-1)^{G_{\mathrm{p}}}=0$ by results of Tate: let $L / k_{\mathrm{p}}$ be a finite extension such that $B$ has good reduction over $L$, that is, extends to an abelian scheme $\mathcal{B}$ over $\mathcal{O}_{L}$. Let $\mathcal{B}(p)$ be the associated $p$-divisible group over $\mathcal{O}_{L}$. Then we have

$$
\begin{aligned}
T_{p}(B)(-1)^{G_{L}} & =\operatorname{Hom}_{G_{L}}\left(T_{p} \mathbb{G}_{m}, T_{p} B\right) \\
& =\operatorname{Hom}_{\mathcal{O}_{L}}\left(\mathbb{G}_{m}(p), \mathcal{B}(p)\right) \quad[\text { Ta2, Theorem 3] } \\
& =\operatorname{Hom}_{\mathcal{O}_{L}}\left(\mathcal{B}(p)^{D}, \mathbb{Q}_{p} / \mathbb{Z}_{p}\right)=0 .
\end{aligned}
$$

Here $\mathcal{B}(p)^{D}=\operatorname{Hom}\left(\mathcal{B}(p), G_{m}\right)$ is the Cartier dual of $\mathcal{B}(p)$, which is connected, since it is the $p$-divisible group of the dual abelian scheme $\mathcal{B}^{\prime}$. We conclude as above

$$
T_{p}(A)(-1)^{G_{\mathfrak{p}}}=T_{p}(T)(-1)^{G_{\mathfrak{p}}}=\operatorname{Hom}_{k_{\mathrm{p}}}\left(G_{m}, T\right) \underset{\mathbb{Z}}{\mathbb{Z}_{p}}
$$

If $A$ has semi-stable reduction, then $X_{*}(T) \cong X_{*}\left(T_{s}\right)$ (isomorphism of $G_{\mathfrak{p}}$-modules via $\left.G_{\mathfrak{p}} \rightarrow \operatorname{Gal}(\overline{\kappa(\mathfrak{p})} / \kappa(\mathfrak{p}))=: \Gamma\right)$ by construction of $T$, and $\operatorname{rank} X_{*}\left(T_{s}\right)^{\Gamma}=\operatorname{dim} T_{s}^{0}$ by definition.

Corollary 7 . a) $H^{2}\left(G_{k}, H^{1}\left(\bar{X}, Q_{\ell} / \mathbb{Z}_{\ell}(r+1)\right)\right)=H^{2}\left(G_{k}, A_{\ell \infty}(r)\right)=0=$ $H^{2}\left(G_{\mathfrak{p}}, A_{\ell \infty}(r)\right)$ for $r \neq 1$.

b) Let $S_{0}$ be the finite set of places, where $A$ does not have potentially good reduction. Then

$$
\begin{aligned}
& H^{2}\left(G_{k}, A_{\ell \infty}(1)\right) \cong \bigoplus_{\mathfrak{p} \in S_{0}} H^{2}\left(G_{\mathfrak{p}}, A_{\ell \infty}(1)\right) \\
& \quad \cong \bigoplus_{\mathfrak{p} \in S_{0}} A_{\ell \infty}\left(k_{\mathfrak{p}}\right)_{G_{\mathfrak{p}}} \cong \bigoplus_{\mathfrak{p} \in S_{0}} X_{*}\left(T_{\mathfrak{p}}^{\prime}\right)_{k_{\mathfrak{p}}}^{*},
\end{aligned}
$$

where $T_{\mathfrak{p}}^{\prime}$ is formed as above, but for the dual abelian variety $A^{\prime}$ (i.e., $T_{\mathfrak{p}}^{\prime}$ is the maximal torus of the Raynaud group scheme $\left.\left(A^{\prime} \times{ }_{k} k_{\mathfrak{p}}\right)^{\natural 0}\right)$.

Proof: This is clear from Theorem 4, Theorem 3 and the local duality

$$
H^{2}\left(G_{\mathfrak{p}}, A_{\ell \infty}(1)\right) \cong\left(T_{\ell} A^{\prime}(-1)^{G_{\mathfrak{p}}}\right)^{*}
$$


coming from the perfect Weil pairings $A_{\ell^{r}}(\bar{k}) \times A_{\ell^{r}}^{\prime}(\bar{k}) \rightarrow \mu_{\ell^{r}}$.

REMARK 8: From the above, we see that $H^{2}\left(G_{k}, A_{\ell \infty}(1)\right)$ may well be non-zero. Hence the same is true for the group $H^{2}\left(G_{S}, A_{\ell \infty}(1)\right)$ mapping onto it (cf. Lemma 5). Next we explain why $H^{2}\left(G_{S}, A_{\ell^{\infty}}\right)$ can be non-zero (compare Example 2): The Kummer sequences $0 \rightarrow A_{\ell^{\nu}} \rightarrow A \stackrel{\ell^{\nu}}{\longrightarrow} A \rightarrow 0$ induce an exact sequence

$$
0 \rightarrow A(k) \otimes \mathbb{Z}_{\ell} \rightarrow H^{1}\left(G_{k}, T_{\ell} A\right) \rightarrow T_{\ell} H^{1}(k, A) \rightarrow 0,
$$

where we have used the fact that $A(k)$ is a finitely generated group by the theorem of Mordell-Weil. We obtain

$$
\operatorname{dim}_{Q_{\ell}} H^{1}\left(G_{S}, V_{\ell} A\right)=\operatorname{dim}_{Q_{\ell}} H^{1}\left(G_{k}, V_{\ell} A\right) \geq \operatorname{rank}_{\mathbb{Z}} A(k) .
$$

On the other hand, we compute by Lemma 2

$$
\chi\left(G_{S}, V_{\ell} A\right)=\chi_{1,1}(X)=-[k: \mathbb{Q}] \cdot \operatorname{dim} A,
$$

since the $\mathbf{R}$-Hodge structure $H^{1}\left(X \times_{\mathbb{Q}} \mathbb{R}, \mathbb{R}\right)$ is of type $\{(0,1),(1,0)\}$ and thus an induced module for $F_{\infty}$. Hence $\operatorname{dim}_{Q_{\ell}} H^{2}\left(G_{S}, V_{\ell} A\right) \geq \operatorname{rank}_{\mathbb{Z}} A(k)-[k$ : Q] $\operatorname{dim} A$, and this number can be strictly positive (there are elliptic curves over $\mathbb{Q}$ with Mordell-Weil rank $>1)$.

This also shows that $\alpha_{2, S}\left(V_{\ell}(A)\right)$ can have a non-zero kernel, since its target is zero by Corollary $5 \mathrm{a})$; compare the exception in Question $2\left(V_{\ell}(A)\right.$ has weight -1).

As explained, these counterexamples concern the case $(i, n)=(1,1)$, but they may also apply to the case $i=2 n-1$, since $H^{2 n-1}\left(\bar{X}, \mathbb{Z}_{\ell}(n)\right)$ may contain the Tate module of an abelian variety, namely the "abelian part" of the $n$-th intermediate Jacobian, compare [Bl].

REMARK 9: Consider the exact sequence

$$
\cdots \rightarrow \underset{\mathfrak{p} \notin S}{\bigoplus} A_{\ell \infty}(r-1)^{G_{\mathrm{p}}} \stackrel{\delta}{\rightarrow} H^{2}\left(G_{S}, A_{\ell \infty}(r)\right) \stackrel{\inf }{\rightarrow} H^{2}\left(G_{k}, A_{\ell \infty}(r)\right) \rightarrow 0
$$

(cf. (13)). Surprisingly, the finite groups $A_{\ell \infty}(r-1)^{G_{\mathfrak{p}}}(\mathfrak{p} \notin S)$ can add up to an infinitely divisible group via $\delta$ for $r=0$ (can one describe this "adding up" in a precise way?). Conjecture 1 claims that this cannot happen for $r \neq 0,1$, and Question 2 asks whether this can happen for $r=1$ (compare Remark 6). 
In the end, we remark that the Iwasawa theoretic question about $Z_{1}=$ $H^{2}\left(H_{S}, H^{1}\left(\bar{X}, \mathbf{Q}_{\ell} / \mathbb{Z}_{\ell}\right)\right)^{*}$ (cf. $\left.\S 3\right)$ is related to Mazur's conjecture [Ma1, p. 184]. In fact, let $A$ be an abelian variety over $k$, let $\ell$ be a prime of

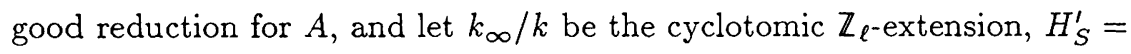
$\operatorname{Gal}\left(k_{S} / k_{\infty}\right)$ etc. as in $\S 3$. Then Mazur's conjecture, as generalized to supersingular primes by Schneider [Sch3, p. 348], says that $H_{\text {fppf }}^{2}\left(\mathcal{O}_{\infty}, \mathcal{A}(\ell)\right)=$ 0 , where $\mathcal{O}_{\infty}$ is the ring of integers in $k_{\infty}, \mathcal{A}$ is the Néron model of $A$, and $\mathcal{A}(\ell)$ is the associated $\ell$-divisible group, regarded as a sheaf for the $f p p f$-topology.

LEMma 14. Assume that the conjecture of Mazur-Schneider is true for $A$ and $\ell$, and let $S$ be a set of places of $k$ containing all places above $\ell \cdot \infty$, and all primes where $A$ has bad reduction. Then $H^{2}\left(H_{S}^{\prime}, A_{\ell \infty}\right)=0$ (i.e., by (21) and Lemma $8, H^{2}\left(G_{S}, A_{\ell \infty}(r)\right)=0$ for almost all $\left.r \equiv 0 \bmod \left[k\left(\mu_{\ell}\right): k\right]\right)$.

Proof: Let $\mathcal{O}_{S, \infty}$ be the ring of $S$-integers in $k_{\infty}$. It suffices to show the surjectivity of the restriction

$$
H_{f p p f}^{2}\left(\mathcal{O}_{\infty}, \mathcal{A}(\ell)\right) \rightarrow H_{f p p f}^{2}\left(\mathcal{O}_{S, \infty}, \mathcal{A}(\ell)\right)
$$

since there are canonical isomorphisms

$$
H_{f p p f}^{2}\left(\mathcal{O}_{S, \infty}, \mathcal{A}(\ell) \cong H_{\text {ét }}^{2}\left(\mathcal{O}_{S, \infty}, \mathcal{A}(\ell)\right) \cong H^{2}\left(H_{S}^{\prime}, A_{\ell \infty}\right)\right.
$$

Indeed, the first one holds, since $\mathcal{A}(\ell)$ is étale over $\mathcal{O}_{S, \infty}$, and the second one follows by the same arguments as (16). For the surjectivity of (53), it suffices to show

$$
H_{f p p f, \mathfrak{P}}^{3}\left(\mathcal{O}_{\infty}, \mathcal{A}(\ell)\right)=0 \text { for } \mathfrak{P} \in \operatorname{Spec} \mathcal{O}_{\infty} \backslash \operatorname{Spec} \mathcal{O}_{S, \infty}
$$

and for this we may pass to the Henselization $\tilde{\mathcal{O}}_{\infty, \mathfrak{P}}$ of $\mathcal{O}_{\infty}$ at $\mathfrak{P}$.

If $\mathfrak{P} \mid \ell$, then the claim follows from [Sch2, Lemma 5], while for $\mathfrak{P} \nmid \ell$ we may again pass to étale cohomology and use the exact sequence

$$
\cdots \rightarrow H^{\nu-1}\left(\tilde{k}_{\infty, \mathfrak{P}}, A_{\ell \infty}\right) \rightarrow H_{\text {èt }, \mathfrak{p}}^{\nu}\left(\tilde{\mathcal{O}}_{\infty, \mathfrak{P}}, \mathcal{A}(\ell)\right) \rightarrow H_{\text {èt }}^{\nu}\left(\tilde{\mathcal{O}}_{\infty, \mathfrak{P}}, \mathcal{A}(\ell)\right) \rightarrow \ldots
$$

where $\tilde{k}_{\infty, \mathfrak{P}}$ is the field of fractions of $\tilde{\mathcal{O}}_{\infty, \mathfrak{P}}$, and $H_{\text {ét }}^{\nu}\left(\tilde{\mathcal{O}}_{\infty, \mathfrak{P}}, \mathcal{A}(\ell)\right) \cong$ $H^{\nu}\left(\kappa(\mathfrak{P}), \mathcal{A}(\ell) \times_{\mathcal{O}_{\infty}} \kappa(\mathfrak{P})\right)$ for the residue field $\kappa(\mathfrak{P})$ of $\mathfrak{P}[\mathrm{Mi1}$, III 3.11]. But $c d_{\ell}(\kappa(\mathfrak{P}))=0$ since $\kappa(\mathfrak{P})$ contains all $\ell$-power roots of unity, and $c d_{\ell}\left(\tilde{k}_{\infty, \mathfrak{P}}\right) \leq 1$ for the same reason. 


\section{REFERENCES}

[Be1] A. Beilinson, Higher regulators and values of L-functions, J. Soviet Math. 30 (1985), 2036-2070.

[Be2] A. Beilinson, Height pairing between algebraic cycles, in "Current Trends in Arithmetical Algebraic Geometry," Comtemporary Mathematics Vol. 67, Amer. Math. Soc., 1987, pp. 1-24.

[BO] P. Berthelot and A. Ogus, F-isocrystals and DeRham cohomology I, Invent. Math. 72 (1987), 159-199.

[Blj S. Bloch, Algebraic cycles and vaiues of L-functions II, Duke Math. J. 52 (1985), 379-397.

[Br] A. Brumer, Galois groups of extensions of number fields with given ramification, Mich. Math. J. 13 (1966), 33-40.

[C] H. Carayol, Sur les représentations L-adiques associées aux formes modulaires de Hilbert, Ann. Sci. École Norm. Sup. (4) 19 (1986), 409-468.

[ChE] C. Chevalley and S. Eilenberg, Colomology theory of Lie groups and Lie algebras, Trans. Amer. Math. Soc. 63 (1948), 85-124.

[D1] P. Deligne, Théorie de Hodge I, in "Actes ICM Nice," t. I, Gauthier-Villars, 1970, pp. 425-430.

[D2] P. Deligne, Formes modulaires et représentations l-adiques, Sém. Bourbaki, exp. 355. Lecture Notes in Math. 179, Springer-Verlag, 1971.

[D3] P. Delinge, Valeurs de fonctions $L$ et périodes d'intégrales, Proc. Symp. Pure Math. 33 (1979), 313-346.

[D4] P. Deligne, La conjecture de Weil II, Publ. Math. IHES 52 (1981).

[D5] P. Deligne, letter to Soulé, January 20, 1985.

[DF] W. Dwyer and E. Friedlander, Étale $K$-theory and arithmetic, Trans. Amer. Math. Soc. 292 (1985), 247-280.

[Fa1] G. Faltings, $p$-adic Hodge theory, Jour. AMS 1 (1988).

[Fa2] G. Faltings, Lecture at Wuppertal, 1987.

[Fo1] J. M. Fontaine, Modules Galoisiens, modules filtrés, et anneaux de Barsotti-Tate, Astérisque 65 (1979), 3-80.

[Fo2] J. M. Fontaine, Sur certains types de représentations $p$-adiques du groupe de Galois d'un corps local: constructions d'un anneau de Barsotti-Tate, Ann. Math. 115 (1982), 529-577.

[FL] J. M. Fontaine and G. Laffaille, Construction de représentations p-adiques, Ann. Sci. École Norm. Sup. (4) 15 (1982), 547-608.

[FM] J. M. Fontaine and W. Messing, $p$-adic periods and $p$-adic étale cohomology, in "Current Trends in Arithmetical Algebraic Geometry," Comtemporary Mathematics Vol. 67, Amer. Math. Soc., 1987, pp. 179-207.

[Gr] A. Grothendieck, Groupes de Barsotti-Tate et cristaux, in "Actes ICM Nice," t. I, Gauthier-Villars, 1970, pp. 431-436.

[J1] U. Jannsen, Continuous étale cohomology, Math. Ann. 280 (1988), 207-245.

[J2] U. Jannsen, Deligne homology, Hodge-D-conjecture, and motives, in "Beilinson's Conjectures on Special Values of $L$-functions," Perspectives in Mathematics, vol. 4 , Academic Press, 1988, pp. 305-372.

[J3] U. Jannsen, "Mixed Motives and Algebraic $K$-Theory," in preparation.

[KM] N. Katz and W. Messing, Some consequences of the Riemann hypothesis for varieties over finite fields, Invent. Math. 23 (1974), 73-77.

[L] M. Lazard, Groupes Analytiques p-Adiques, Publ. Math. IHES 26 (1965).

[Ma1] B. Mazur, Rational points of abelian varieties with values in towers of number fields, Invent. Math. 18 (1972), 183-266. 
[Ma2] B. Mazur, Modular curves and elliptic curves, Lecture at Harvard Univ., Spring 1984.

[MTT] B. Mazur, J. Tate and J. Teitelbaum, On p-adic analogues of the conjectures of Birch and Swinnerton-Dyer, Invent. Math. 84 (1986), 1-48.

[Mi1] J. S. Milne, "Étale Cohomology," Princeton Univ. Press, 1980.

[Mi2] J. S. Milne, "Arithmetic Duality Theorems," Perspectives in Mathematics, Vol. 1, Academic Press, Boston, 1986.

[Neu] O. Neumann, On p-closed algebraic number fields with restricted ramification, Math. USSR Izv. 9 (1975), 243-254.

[Qui] D. Quillen, Higher algebraic K-theory, in "Proc. ICM Vancouver 1974," Canadian Math. Congress, 1975.

[RZ] M. Rapoport and Th. Zink, Über die lokale Zetafunktion von Shimuravarietäten. Monodromiefiltration und verschwindende Zyklen in ungleicher Charakteristik, Invent. Math. 68 (1982), 21-101.

[Sch1] P. Schneider, Über gewisse Galoiscohomologiegruppen, Math. Z. 168 (1979), 181-205.

[Sch2] P. Schneider, Iwasawa L-functions of varieties over algebraic number fields: A first approach, Invent. Math. 71 (1983), 251-293.

[Sch3] P. Schneider, $p$-adic height pairings II, Invent. Math. 79 (1985), 329-374.

[Se1] J.-P. Serre, "Cohomologie Galoisienne," Lecture Notes in Math. 5, Springer-Verlag, 1973.

[Se2] J.-P. Serre, Sur les groupes de congruence de variétés abéliennes I, Izv. Akad. Nauk SSSR, Sér. Mat. 28 (1964), 3-20.

[Se3] J.-P. Serre, Sur les groupes de congruences des variétés abéliennes II, Izv. Akad. Nauk SSSR, Sér. Mat. 35 (1971), 731-737.

[Se4] J.-P. Serre, "CEuvres - Collected Papers," Springer-Verlag, Berlin, 1986.

[Sou1] C. Soulé, $K$-théorie des anneaux d'entiers de corps de nombres et cohomologie étale, Invent. Math. 55 (1979), 251-295.

[Sou2] C. Soulé, On higher p-adic regulators, in "Algebraic $K$-Theory, Evanston 1980," Lecture Notes in Math. 854, Springer-Verlag, Berlin, 1981, pp. 372-401.

[Sou3] C. Soulé, Operations on étale $K$-theory, in "Algebraic $K$-Theory, Oberwolfach 1980," Lecture Notes in Math. 966, Springer-Verlag, Berlin, 1982, pp. 271-303.

[Sou4] C. Soulé, The rank of étale cohomology of varieties over p-adic or number fields, Comp. Math. 53 (1984), 113-131.

[Sou5] C. Soulé, p-adic K-theory of elliptic curves, Duke Math. J. 54 (1987), 249-269.

[Ta1] J. Tate, Duality theorems in Galois cohomology over number fields, in "Proc. ICM, Stockholm 1962," Institut Mittag-Leffler, 1963, pp. 288-295.

[Ta2] J. Tate, p-divisible groups, in "Proceedings of a Conference on Local Fields, Driebergen 1966," Springer-Verlag, 1967, pp. 153-183.

[Ta3] J. Tate, Relations between $K_{2}$ and Galois cohomology, Invent. Math. 36 (1976), 257-274.

[Th] R. Thomason, Bott stability in algebraic K-theory, in "Applications of Algebraic $K$-Theory to Algebraic Geometry and Number Theory, Part I," Contemporary Math., Vol. 55, Amer. Math. Soc., 1986, pp. 389-406.

[Wak] R. Wake, Phantom points on abelian varieties, preprint.

[Wa1] L. Washington, "Introduction to Cyclotomic Fields," Graduate Texts in Math., Vol. 83, Springer-Verlag, 1982.

[Wa2] L. Washington, Zeroes of p-adic L-functions, in "Sém. de Théorie des Nombres, Paris 1980-81," Birkhäuser, 1982, pp. 337-357.

[Wi1] K. Wingberg, Duality theorems for $\Gamma$-extensions of algebraic number fields, Comp. Math. 55 (1985), 333-381. 
[Wi2] K. Wingberg, On the étale $K$-theory of an elliptic curve with complex multiplication for regular primes, preprint.

[SGA 7 1] A. Grothendieck, et al., "Groupes de Monodromie en Géométrie Algébrique," Lecture Notes in Math. 288, Springer-Verlag, Berlin, 1972.

FB Mathematik, Universität Regensburg, Universitätstr. 31, 8400 Regensburg, FEDERAL REPUBLIC OF GERMANY 